\title{
Corela
}

Cognition, représentation, langage

HS-7 | 2010

Espace, Préposition, Cognition

\section{Mise en scène, hors-champ et sémantique prépositionnelle. Une modélisation de l'emploi situatif sur+ville}

Patricia C. Hernández

\section{OpenEdition}

Journals

Édition électronique

URL : http://journals.openedition.org/corela/882

DOI : $10.4000 /$ corela.882

ISSN : 1638-573X

Éditeur

Cercle linguistique du Centre et de l'Ouest - CerLICO

Référence électronique

Patricia C. Hernández, « Mise en scène, hors-champ et sémantique prépositionnelle. Une modélisation de l'emploi situatif sur+ville», Corela [En ligne], HS-7 | 2010, mis en ligne le 31 mai 2010, consulté le 30 avril 2019. URL : http://journals.openedition.org/corela/882 ; DOI : 10.4000/corela.882

Ce document a été généré automatiquement le 30 avril 2019.

\section{(c) (i) (2)(2)}

Corela - cognition, représentation, langage est mis à disposition selon les termes de la licence Creative Commons Attribution - Pas d'Utilisation Commerciale - Partage dans les Mêmes Conditions 4.0 International. 


\title{
Mise en scène, hors-champ et sémantique prépositionnelle. Une modélisation de l'emploi situatif sur +ville
}

\author{
Patricia C. Hernández
}

\section{Introduction}

1 Cet article rend un modeste hommage à Claude Vandeloise dont les recherches ont profondément inspiré notre travail (2006). Plusieurs concepts développés par le linguiste sous-tendent les pages qui suivent. D'abord, l'idée d'un espace à l'échelle humaine : dans sa mise en mots de la spatialité, l'homme verbalise sa conceptualisation de l'univers environnant avec des perspectives changeantes et multiples, créativité que l'auteur de L'espace en français conçoit comme un aspect essentiel du langage.

2 Autre notion fondamentale, le rôle des scénarios établis pour la construction et l'interprétation des scènes spatiales: l'expérience quotidienne et les rituels sociaux tissent l'expression des relations spatiales sur la base d'un savoir partagé, trame sur laquelle s'inscrit le discours.

3 Nous devons également à Claude Vandeloise un concept de base pour l'étude du sémantisme des prépositions dites spatiales : le contraste localisation / configuration que nous concevons ici comme un continuum donnant lieu à des coefficients variables selon le contexte.

4 Enfin, certains principes comme la relation fonctionnelle entre la cible, entité à localiser, et le site, entité localisatrice, le principe d'anticipation et les localisations emboitées trouveront leur place dans cette étude qui vise à rendre compte d'une évolution récente dans le positionnement des relateurs français. 
5 En effet, une description du paysage prépositionnel du français actuel ne saurait ignorer l'essor spectaculaire de la préposition sur. Marqueur de superposition selon la vision traditionnelle, ce terme empiète désormais sur le terrain d'autres relateurs qu'il tend à déplacer. C'est le cas de à qui apparaît concurrencé par sur même pour des emplois reconnus comme typiquement localisateurs. Plutôt que de considérer cette alternance comme un simple phénomène de flottement stylistique, nous décelons, dans cet emploi innovant, l'émergence d'un rapport sémantique original, certes instable, caractérisé par une intrication entre domaines linguistiques.

6 Notre démarche théorique, en deux temps, abordera, dans un premier moment, le sémantisme des prépositions sur et à en emploi localisateur, notamment devant les noms de ville. Nous y mettrons à contribution certains principes distinctifs élaborés par Claude Vandeloise et ferons l'état des lieux du traitement de ce phénomène particulier. Dans un second temps, sera traitée la mise en scène de la spatialité : la notion de scène verbale (Victorri 1997, 1999) fournira le cadre théorique de notre analyse des phénomènes de monstration selon un certain cadrage de la scène spatiale.

7 Ces deux volets se rejoindront dans un troisième temps, lorsque nous traiterons l'emploi sur+ville avec des verbes situatifs comme un déclencheur d'inférences susceptible d'être modelisé à l'aide de la notion de hors champ absolu (Deleuze 1983).

\section{L'émergence de l'emploi situatif sur+ville}

\subsection{Différence entre à et sur pour l'expression de la spatialité}

\subsubsection{Localisation vs configuration}

8 En matière de sémantique prépositionnelle, la littérature de spécialité fait traditionnellement appel à une distinction fondamentale sur la base de la «substance sémantique " renfermée par ces éléments de relation et identifie, d'une part, des prépositions casuelles, vides, abstraites, incolores - synsémantiques selon Pierre Cadiot (1997a: 130) - et, d'autre part, non-casuelles, pleines, colorées - autosémantiques -. Entre les deux, apparaît parfois une catégorie intermédiaire, celle des prépositions appelées semi-casuelles (De Boer 1926), à demi-vides (Brunot et Bruneau 1933) ou mixtes (Cadiot 1997a). Ce qui pourrait être figuré sous la forme d'un continuum entre deux pôles : un pôle de vacuité occupé par la préposition de, suivie de à et en, et un pôle de "complétude sémantique » où l'on trouve des prépositions comme contre, parmi ou vers, en passant par une zone médiane, celle des relateurs par, pour, avec, dans, sur, sous, qui cumulent sens concret (généralement spatial) et emplois dits abstraits (domaines temporel et notionnel).

9 Ce clivage entre termes vides et termes pleins prend souvent la forme d'une bipolarisation entre abstraction et matérialité, tension qui sous-tend l'étude contrastive de certaines prépositions, en l'occurrence celle du binôme à - sur. C'est ainsi que la préposition à, considérée comme "grammaticale » en vertu de son origine latine, de la multiplicité de ses emplois, des commutations possibles auxquelles elle se prête et de la diversité de traductions qu'elle semble susciter, est unanimement rangée près du pôle de l'abstraction. En revanche, sur, qui semblerait, à première vue, s'orienter vers le pôle des particules à sémantisme plein, ne fait pas l'objet d'un tel consensus. En effet, la préposition sur est considérée, selon les différents auteurs, comme étant colorée (SpangHanssen 1963), pleine (Ruwet 1982), à demi-vide (Brunot et Bruneau 1933), mixte (Cadiot 
1997a), incolore quoique «moins décolorée » que les autres (Wartburg et Zumthor 1947), incolore « de deuxième génération » (Cadiot 1997a), «blanchie » (Melis 2003). Autrement dit, la catégorisation du sémantisme de sur s'avère être moins stabilisée peut-être en raison des récentes extensions d'emploi qui semblent suggérer une évolution en cours.

Le domaine de la spatialité reproduit la polarisation abstraction-concrétude sous la forme d'une distinction de base entre localisation et configuration (Vandeloise 1987, 1988, 1990). Relation fonctionnelle, la localisation apparaît comme un repérage abstrait dépendant de la spécificité du site et de la connaissance partagée, à la différence de la configuration qui représente une opération de type plus descriptif, acceptant des détails contingents et moins dépendante des scénarios établis. Le linguiste $(1990: 170)$ souligne le rôle déterminant des rituels sociaux dans la sélection des prépositions : alors que la préposition à intervient en général dans des opérations abstraites de localisation jouant sur le savoir préexistant, sur semble participer à la construction de configurations de type descriptif moins dépendantes des rituels institués.

11 Ainsi, derrière la distinction vandeloisienne entre localisation et configuration, pointe la tension entre inférence pour les prépositions incolores et codage pour les prépositions colorées (Cadiot 1997a: 133). En effet, les marqueurs a-descriptifs comme à, sémantiquement moins denses, sollicitent plus fortement les mécanismes inférentiels dans les processus interprétatifs, à l'opposé des termes configurationnels, par exemple sur en emploi spatial, qui, en général, semblent coder la spatialité de manière plus explicite. La préposition à serait donc située dans la sphère de l'abstraction associée à la localisation et à l'inférence tandis que sur demeurerait dans le domaine coloré de la matérialité, la configuration et le codage descriptif. Or il faut noter que sur connaît de nouveaux emplois qui semblent répondre, à nos yeux, à un déplacement graduel du terme sur l'axe du sémantisme prépositionnel : partant de la zone médiane de la matérialité et de la configuration spatiale codée, sur semble se rapprocher, dans certains emplois récents, du pôle de l'abstraction mettant en jeu des processus inférentiels.

Il va de soi que ce repositionnement induit un portrait plus nuancé du relateur qui semble posséder des coefficients de configurationnalité variables. Avant d'analyser un cas particulier de cette évolution, nous évoquerons rapidement les caractéristiques saillantes des deux prépositions dans leur emploi dit spatial.

\subsubsection{L'a-descriptivité de à}

13 Dans ses emplois dynamiques ou statiques, la préposition à semble viser la partie terminale d'un mouvement d'approche, imprégnant ainsi de ponctualité - certains parlent d'éloignement, voire d'absence du champ visuel (Herskovits 1981, Vandeloise 1988) - l'espace non fractionné et non qualifié qu'elle aide à construire.

Paul va / est à Paris

Le repérage construit par ce marqueur essentiellement économique repose sur la connaissance partagée qui aide à composer une image abstraite, synthèse de l'expérience, évoquant une situation typique inférée au départ d'une routine d'interaction conventionnellement associée au site de référence.

Paul va / est au bureau

Le rôle crucial de ce savoir partagé, à l'œuvre dans le processus inférentiel, transparaît, dans le discours, par la résistance du relateur à l'indétermination (Vandeloise $1988: 129$ ) : 
Paul va / est au bureau

Paul va / est à un bureau et la description cède le pas au repère - Paul est au lit se rapprochant de la formulation situationnelle Paul est alité. D'où la création d'un lieu-fonction (Cadiot 1997b : 220). Condition sine qua non, un rapport fonctionnel nécessairement pertinent comme l'illustre l'exemple proposé par Claude Vandeloise (1988:140):

Le chien est à l'école vs Le chien est dans l'école

L'activation d'un scénario typique peut être déclenchée par toute entité dont la valeur culturelle ou symbolique se trouve cognitivement instituée : institutions possédant un siège physique ou non, objets déterminant une attitude posturale particulière ou événements, rituels sociaux, activités.

Paul est à l'Université

Paul est au piano

Paul est aux sports d'hiver

Ce qui aboutit à la dématérialisation du site. Ainsi, dans les énoncés suivants,

Paul est dans le lit/ Paul est sur le lit vs Paul est au lit

la description construite à l'aide des relateurs dans et sur s'oppose à l'abstraction de la situation décrite par l'énoncé en à : dans ce dernier, le site perd de sa saillance matérielle

effet, à moins d'imaginer un scénario de dessin animé ou de fiction, une telle formulation apparaîtra comme «anormale» puisque, dans la connaissance communément partagée, il n'existe pas d'interaction typique entre un chien et une école.

Dans cette perspective, les emplois spatiaux de la préposition à, évoquant traditionnellement des notions de ponctualité et de directionnalité, se doublent d'une fonction inférentielle apte à activer un scénario typique. Et ce dans le cadre d'une localisation spatiale générique sur la base d'un espace idéalisé sans détails contingents. A preuve, la résistance du relateur à l'ajout de qualifications accidentelles (Vandeloise $1988: 141)$ :

Baudouin est à la caserne

Baudouin est à la caserne dont les barbelés brillent sous la pluie

Baudouin est dans la caserne dont les barbelés brillent sous la pluie

C'est pourquoi nous proposons, pour à, le terme de relateur a-descriptif.

\subsubsection{La configurationnalité de sur}

Les critères généralement évoqués pour la caractérisation de ce marqueur en emploi spatial sont plus ou moins liés à la prégnance de la gravitation, à commencer par la notion de superposition sur l'axe vertical, et de charge; d'où les concepts de support et de contact si répandus dans la littérature.

Porter un fardeau sur ses épaules

23 On peut distinguer, en grandes lignes, deux cas de figure: d'une part, une orientation haut-bas, liée à l'idée de poids, voire à celle de recouvrement du site et, en contrepartie, de visibilité de la cible

La tasse est sur la table

Et, d'autre part, une orientation sur un axe horizontal, activant la notion d'adhérence

Une affiche sur le mur 

certaines configurations spatiales (par exemple, " une affiche sur le mur ») comme étant canoniques voire à les associer à un scénario typique donnant lieu à une simple localisation ("une affiche au mur »). D'où, dans certains cas, l'alternance des deux prépositions. Découlant d'un figement de l'emploi, une telle conventionnalisation atténue la valeur descriptive du relateur configurationnel, ce qui se traduit par la banalisation de l'alternance à / sur dans certains contextes :

Voir une affiche sur le mur / au mur

Être sur la plage / à la plage

L'éventuel enracinement de cette alternance prépositionnelle peut conduire à un flottement, dans le discours, entre emplois configurationnels et localisateurs, avec un estompage progressif de la matérialité de l'espace évoqué par les énoncés en sur. En effet, dans certains énoncés, par exemple

Les enfants jouent sur le trottoir

33 la région d'interaction se trouve « investie dans la construction d'espaces fonctionnels plutôt que physiques» (Cadiot et Visetti $2001: 26)$. Ce qui revient à étendre à l'interprétation de certains énoncés en sur, la notion de lieu-fonction, évoquée pour à, localisation caractérisée par une relation fonctionnelle entre la cible et un site idéalisé. 

spatiale contribue à atténuer la valeur descriptive de l'énoncé, en vertu d'un principe d'économie cognitive pour les situations typiques :

L'affiche est sur le mur / L'affiche est au mur

La frontière entre localisation et configuration, du moins pour l'analyse de certains énoncés, s'avère donc moins nette qu'on ne pourrait le croire puisque sur semble jouer sur différents registres de configurationnalité : plus la scène évoque une configuration particulière, plus l'emploi gagnera en force descriptive ; en revanche, les configurations instituées comme canoniques amenuisent cette force descriptive et tendent vers le simple repérage. C'est ce qu'illustrent les formulations suivantes :

Le chien est sur le lit

La tasse est sur la table

Paul est sur la plage

Dans la scène du chien sur le lit, comme configuration particulière s'opposant, par exemple, à sous le lit, la préposition conserve une valeur descriptive marquée en vertu d'une certaine instabilité due à l'ancrage insuffisant de la relation spatiale. En revanche, dans la tasse est sur la table, la configurationnalité du relateur est moins saillante car il évoque une interaction typique instituée par l'expérience quotidienne. Enfin, l'énoncé Paul est sur la plage met en scène une spatialisation moins descriptive où la configuration, amoindrie, se rapproche de la simple localisation sur un support ${ }^{1}$.

En même temps, moins la routine sociale et la mémoire linguistique sont instituées, moins l'emploi de à, préposition supposant des connaissances partagées, semble acceptable. Ainsi semblent le suggérer les exemples suivants (Vandeloise $1990: 172$ ) :

Le tableau est au mur / *Le tableau est à la paroi

Le chapeau est au porte-manteau / *Le chapeau est à la branche

L'alternance à / sur se laisserait donc décrire selon deux cas de figure :

(1) un partage des tâches pour les scènes particulières non associées à des figements linguistiques. Dans ce cas, la localisation a-descriptive étant insuffisante à assurer un repère pertinent, seule la configurationnalité de sur aide à construire la scène spatiale :

L'affiche est sur la paroi / *L'affiche est à la paroi

(2) des emplois concurrents lorsque l'ancrage cognitif et linguistique de la relation

la base de cette alternance, l'adoucissement de la spécificité configurationnelle de sur ${ }^{2}$, préposition qui apparaît, de nos jours, en concurrence non seulement avec à mais aussi avec vers (se diriger vers / sur Paris) et dans (dans / sur le journal) ; extension d'emplois qui rapproche le marqueur configurationnel du pôle de l'abstraction.

41 Ce phénomène est attribué par Goyens, Lamiroy et Melis (2002:298) à l'érosion de la préposition à, marqueur abstrait dont les emplois locatifs seraient en nette régression. Pour les auteurs, à se trouve sévèrement concurrencé par sur en emploi directionnel et par dans en emploi positionnel. Or le seul repli de à ne saurait suffire, nous semble-t-il, à décrire un phénomène complexe mettant en jeu la place respective de plusieurs prépositions : si à perd du terrain, c'est devant l'avancée spectaculaire de sur, essor qui dépasse celui de dans. En outre, les énoncés en à n'alternent pas systématiquement avec des formulations en sur pour les énoncés directionnels et en dans pour les emplois statiques. Ainsi, Je vais/suis à Paris ne devient pas nécessairement Je vais sur Paris/Je suis dans Paris (formulation possible, bien sûr, mais comportant une variation sémantique). Qui plus est, Je suis à Paris se trouve souvent supplanté non pas par Je suis dans Paris mais par Je suis...sur Paris. Ce qui prouve que, loin de se cantonner aux emplois directionnels, 
sur investit aussi les énoncés situatifs. Exemple emblématique de cette déferlante, le flottement $\grave{a}$ / sur dans les formulations suivies d'un nom de ville, expressions où à était naguère seul maître à bord, comme le signale Denis Slakta (1990: 102-103) qui relève, entre autres, l'alternance Je travaille à / sur Paris.Les énoncés en sur+ville constituent, on le voit, une nouveauté, repérable surtout dans les pratiques langagières quotidiennes.

\subsection{La concurrence à - surt ville}

\subsubsection{L'alternance prépositionnelle devant les noms de ville}

Phénomène récent, l'alternance $\grave{a}$-sur devant un nom de ville ${ }^{3}$ ne fait que depuis peu l'objet d'un traitement systématique. Etudes linguistiques, grammaires et dictionnaires sont à ce sujet fort éclairants qui envisagent, pour la plupart, des contrastes $\grave{a}$ - dans, $\grave{a}$ - en , voire $\grave{a}$ - pour alors que le contraste $\grave{a}$ - sur n'est que rarement explicité, sur+ville faisant l'objet d'un traitement indépendant, souvent en concurrence avec la préposition vers.

Nous passerons rapidement en revue certaines alternances traitées dans la bibliographie de spécialité. Pour cela, nous distinguerons, d'une part, des flottements entre la préposition à et des marqueurs considérés comme évoquant un emploi dynamique (à vs jusqu'à/en, pour, vers) et, d'autre part, des alternances avec des marqueurs susceptibles d'emplois situatifs mais aussi dynamiques, en vertu du principe d'anticipation (en - dans). Après la description de l'alternance sur - vers pour le marquage de la directionnalité, nous aborderons le traitement du binôme à - sur + ville.

\subsubsection{1. à - jusqu'à/en - pour - vers + ville}

Les quatre prépositions sont groupées par Patrick Charaudeau (1992: 416-418) comme participant à l'évocation d'un mouvement d'approche en combinaison avec des verbes de déplacement. Une distinction majeure sera l'atteinte du point d'arrivée, induite par les emplois de à et jusque $+\grave{a} / e n$, passée sous silence dans le cas de vers et pour qui n'explicitent pas si la destination est atteinte. L'approche du linguiste ne comporte pas de contraste prépositionnel binaire.

Cependant, ces relateurs ont parfois fait l'objet de traitement par paires : le couple $\grave{\boldsymbol{a}}$ jusqu'à+ville apparaît chez Pottier (1962 : 206), dans la section consacrée à la préposition $\grave{a}$. La formulation Aller jusqu'à Paris suppose l'atteinte accompagnée, jusque introduisant une visée accompagnante.

La paire $\grave{a}$ - vers +ville est généralement traitée sur la base d'une différence sémantique entre l'indication du terme du déplacement (Je vais à Paris) et la direction du mouvement ( Je vais vers Paris). - Avec des verbes situatifs, la distinction tient, selon G. Gougenheim (1938 : 302), à une question de précision : la préposition à indique un point précis alors que vers semble évoquer une région de manière plus vague : "Il demeure à Châteauroux » et « Il habite une jolie maison de campagne vers Châteauroux » (G. Sand, Mauprat, p. 7).

Pour ce qui est du binôme $\grave{a}$ - pour +ville, la préposition $\grave{a}$, en tant que relateur directionnel pour la construction d'énoncés dynamiques, est présentée comme susceptible de remplacer pour dans la tournure partir pour+ville (Brunot 1953 : 432-433, Vandeloise 1987 : 103, Le Grand Robert article à). Pierre Cadiot (1991: 49-50) décrit cette alternance prépositionnelle avec des verbes directionnels : tandis que $\grave{a}+v i l l e$ anticipe sur 
l'effectuation d'une trajectoire avec atteinte du point d'arrivée, pour +ville n'implique pas que la destination est atteinte.

\subsubsection{2. à - en - dans + ville} de ville :

La préposition à - "préposition usuelle devant les noms de villes non qualifiés », selon Spang-Hanssen (1963: 197) - apparaît, dans tous les cas, avec sa double valeur, dynamique et statique, marquant le «directif » et le locatif, selon Le Bidois et Le Bidois (1971 : 677), double vocation qui apparaît déjà dans le Littré sous la forme d'une valeur de direction (aller à Paris) et de «repos » (résider à Paris). «On dit, est-il précisé, à Paris, à Bordeaux, quand il s'agit de demeure soit fixe soit passagère. Il est à Paris, il réside à Paris, il passera quelques jours à Paris; autrement on peut dire dans : il y a douze cent mille habitants dans Paris ».

L'opposition $\grave{a}$ - dans + ville est traitée en termes de ponctualité (localisation abstraite) vs intériorité et étendue (configuration d'une intériorité bornée) par Gougenheim (1938 : 295), Pottier (1962: 217), Spang-Hanssen (1963: 197), Von Wartburg et Zumthor (1973: 361), Grevisse (1980 : 1183), Colin (1994: 1), Girodet (1997: 7).

Il se trouve à Lyon / il va à Bordeaux / il habite à Paris

«Est-ce que vous ne savez pas que les armées allemandes sont dans Paris?»

Colette, L'Etoile Vesper. p. 27 in Grevisse 1980 : 1183)

Harald Weinrich formulera cette opposition en termes de lieu d'actance vs inclusion dans des limites urbaines (1989: 424).

Le choix de l'une ou de l'autre des prépositions peut aussi être associé à l'emploi de la détermination (Spang-Hanssen 1963 : 198, Le Bidois et Le Bidois 1971 : 717).

à Paris vs dans le Paris d'aujourd'hui

Comme nous l'avons vu, dans peut intervenir dans la formation d'énoncés aussi bien statiques (être dans Paris) que dynamiques (entrer dans Paris) en vertu du principe d'anticipation des prépositions configurationnelles.

La paire $\grave{a}$ - en devant les noms de ville commençant par voyelle (à Avignon vs enAvignon) fait l'objet de remarques d'ordre stylistique ${ }^{5}$ : l'emploi de en est généralement critiqué et considéré comme affecté (Gougenheim 1938: 300, Von Wartburg et Zumthor 1973: 369, Grevisse 1980 : 1186, Colin $1994: 1$, Girodet 1997 : 7, Péchoin et Dauphin $1998: 1$ ).

4 Dans les dictionnaires des difficultés où apparaît fréquemment le trinôme à - en - dans, c'est la préposition à qui est jugée comme étant le relateur par excellence devant les noms

«Les noms de ville se construisent toujours avec la préposition à " (Péchoin et Dauphin $1998: 1$ )

«On emploie normalement à devant un nom de ville » (Girodet $1997: 7$ ) (c'est nous qui soulignons)

\subsubsection{3. sur - vers + ville}

Sur+ville apparaît dans des énoncés dynamiques pour le marquage de la direction (Brunot 1953 : 432-433) et la désignation d'un objectif (TLFi article sur) :

Marcher sur Rome (TLFi)

La ruée sur Calais (Brunot 1953) 
Ainsi, comme relateur directionnel, sur se trouve, devant les noms de ville, souvent en concurrence avec vers (Gougenheim 1938 : 314, Brunot 1953 : 432-433, Wagner et Pinchon 1962 : 488, Girodet 1997 : 741, Péchoin et Dauphin 1998 : 551, Le GrandRobert article sur) :

« En roulant par là, vous allez sur Doullens » (Wagner et Pinchon 1962)

«Une voiture comme celle qui va sur Beaumont » (Balzac cité par Le Grand Robert, article flambant)

«Ce jour-là l'armée, qui venait de gagner la bataille de Ligny, était en pleine marche sur Bruxelles » (Stendhal, La chartreuse de Parme, II, cité par Gougenheim 1938 : 314)

Les dictionnaires des difficultés y voient un emploi abusif et conseillent de s'en tenir à la tournure correcte avec la préposition vers :

Les véhicules se dirigeant vers Lille et non sur Lille (Péchoin et Dauphin 1998 : 551)

Les automobiles se dirigeant sur Paris au lieu du tour correct vers Paris (Girodet

$1997: 741)$

avec à chez G. Gougenheim (1938: 301) dans le cadre de l'opposition binaire envisagée par le linguiste pour l'ensemble des prépositions. Selon l'auteur du Système grammatical de la langue française, les deux relateurs se distinguent en ce que l'emploi de sur est empreint d'une nuance de rapidité : «à marque le terme d'un mouvement, sur, la direction d'un mouvement rapide »

Je vais à Paris vs L'ennemi marcha sur Paris

Le relateur marque aussi l'objectif, le but précis d'une opération, signale Jean Cervoni (1991 : 229) qui, suivant Brøndal, relève l'emploi de sur dans le langage militaire (« il se dirigea sur Amiens »).

En dehors des emplois dynamiques, sur+ville est évoqué, en emploi statique, par Bernard Pottier (1962 : 235) comme correspondant à la «situation par rapport à l'autre côté de la limite, à une certaine distance ", par exemple dans

Je travaille sur Marseille

signale que le complément introduit par sur peut désigner le lieu d'une activité :

Travailler sur le secteur de, sur Lunéville

64

Or, depuis une vingtaine d'années, l'étude comparative fait place à la constatation d'un changement dans le statut quo prépositionnel. Denis Slakta signale, on l'a vu, l'avancée de sur au détriment de à dans des emplois de type situatif, du genre "Je travaille à/sur Paris », extension récente, selon le linguiste : « Les gens de ma génération, souligne-t-il, emploient à et dans; nos enfants, sur " (1990 : 102-103). La séquence sur + ville est condamnée par Jean-Paul Colin (1994: 583) : « Avec un nom de localité, [la préposition sur 
] tend depuis quelques années à assumer des acceptions discutables" (c'est nous qui soulignons). L'auteur du Dictionnaire des difficultés du français cite B. Poirot-Delpech (Le Monde, 21 février 1990) :

«[Sur] est en train de supplanter toutes les prépositions de lieu. Non seulement on rentre sur Paris, mais on travaille sur la capitale à la façon dont les vendeurs, jamais en retard d'une métaphore guerrière, mettent le paquet sur une région, sur un produit».

Et J.-P. Colin de renchérir : «Cet emploi n'ajoute rien à la préposition à. On peut, si l'on veut être précis ( ?) employer le tour dans le secteur de.»

Ce qui appelle deux remarques : d'une part, l'idée que sur se substitue simplement à la préposition à sans ajout sémantique particulier, position qui n'est pas la nôtre, et d'autre part, l'attribution à ce nouvel emploi, paradoxalement, d'une nuance d'indéfinition spatiale (dans le secteur de) par opposition à la ponctualité de à. Ce sera l'avis de certains qui verront dans cet usage une valeur d'approximation.

Dans Grammaire des prépositions (Franckel et Paillard 2007: 72, 95), Denis Paillard traite explicitement l'opposition à / sur devant les noms de ville. Le linguiste y décèle une divergence sémantique: à+ville évoquerait une simple localisation du sujet ou de l'événement tandis que sur+ville (aller sur Paris, travailler sur Paris), plus difficile à définir, dépasse le cadre de la spatialité pour qualifier le sujet. L'auteur traite en parallèle énoncés directionnels et situatifs.

Dans l'alternance aller à / sur Paris, la distinction résiderait, selon le linguiste, dans l'indication du point de destination, pour les énoncés en à, et l'évocation d'une direction et non pas de la destination finale, pour les énoncés en sur. Or une précision s'impose pour la cooccurrence de sur avec les verbes de déplacement. Sur tend à remplacer vers pour des emplois dynamiques (Je vais vers / sur Paris) et à pour des emplois à la fois dynamiques (Je vais à / sur Paris) et statiques (Je suis à / sur Paris). Certains emplois directionnels de sur recouvrent donc potentiellement les prépositions à et vers :

Je vais sur Paris $=$ Je vais $\grave{a} /$ vers Paris

Autrement dit, le contraste à / sur semblerait sémantiquement apparenté à l'opposition à /vers.

Pour ce qui est des verbes statiques, Denis Paillard (2007: 95) souligne le rapport «temporaire» qui unit les deux termes en relation : dans X travaille sur Paris, la cible se trouve en rapport avec la ville le temps de son travail (il habite à Orléans mais il travaille sur Paris), lien qui devient plus complexe avec le verbe être car la relation induite par la préposition semble dépasser la spatialité proprement dite pour qualifier le sujet impliqué dans procès ; ainsi, le sujet sera perçu comme "parisien " pendant la durée du procès (travail, séjour) - Cf. 3.1.

\subsection{L'essor spectaculaire de sur}

71 Pour l'instant peu fréquent en langue écrite (emploi non attesté, par exemple, dans le corpus Frantext et rare dans la presse) l'emploi de sur+ville gagne du terrain à l'oral et s'installe comme alternative aux formulations en à. Pour mesurer l'ampleur du phénomène, nous avons consulté à plusieurs reprises les petites annonces personnelles sur Internet correspondant à un discours spontané avec tous les traits de l'oralité. La préposition sur s'y trouve en cooccurrence non seulement avec des verbes de mouvement 
comme arriver, aller ou venir en emploi congruent avec leur directionnalité, mais aussi avec des verbes considérés comme statiques tels que être, habiter, rester, travailler voire dormir. Voici les résultats d'une recherche, effectuée sur Yahoo France les 8 et 9 mars $2006^{6}$ , sur des énoncés situatifs suivis des syntagmes à Paris / sur Paris en emploi concurrent :

\begin{tabular}{|c|c|c|c|}
\hline \multicolumn{4}{|l|}{ Mars 2006} \\
\hline \multirow{3}{*}{ Verbes cooccurrents } & & & \\
\hline & \multicolumn{2}{|c|}{ Nombre d'occurrences } & \\
\hline & \multicolumn{2}{|l|}{ à Paris } & sur Paris \\
\hline Je suis & 38300 & 5620 & \\
\hline J'habite & 33900 & 804 & \\
\hline Je travaille & 418 & 250 & \\
\hline Je reste & 434 & 133 & \\
\hline Je dors & 14 & 8 & \\
\hline
\end{tabular}

On le voit, à l'exception de habiter majoritairement construit avec à, des verbes généralement statifs rentrent, en nombre élevé, dans des énoncés avec sur. Détail frappant, en cooccurrence avec être, sur l'emporte sur à.

Deux ans plus tard, nous avons refait notre recherche, sur Yahoo France (le 30 mai 2008). Voici les résultats :

\begin{tabular}{|c|c|c|c|}
\hline \multicolumn{4}{|l|}{ Mars 2008} \\
\hline \multirow{3}{*}{ Verbes cooccurrents } & & & \\
\hline & \multicolumn{2}{|c|}{ Nombre d'occurrences } & \\
\hline & \multicolumn{2}{|l|}{ à Paris } & sur Paris \\
\hline Je suis & 170000 & 1110 & 000 \\
\hline J'habite & 103000 & 1260 & \\
\hline Je travaille & 11500 & 2940 & \\
\hline Je reste & 2980 & 929 & \\
\hline Je dors & 86 & 46 & \\
\hline
\end{tabular}


Il ne s'agissait donc pas d'un engouement passager. La préposition sur semble bel et bien installée comme localisateur statique : si on enregistre un certain recul de la fréquence du marqueur avec le verbe être (le taux d'occurrences passe de 59,47 \% en 2006 à 39,50 \% en 2008) et travailler (37,42\% en 2006 contre $20,36 \%$ deux ans plus tard), il faut noter que la tendance se maintient avec les verbes rester (en 2006, $23,45 \%$ contre $23,76 \%$ en 2008) et dormir (36,36 \% et $34,84 \%$ respectivement).

L'ampleur du phénomène mérite que l'on s'y arrête.

76 Remarquons également que cet emploi prépositionnel n'est pas l'apanage exclusif du syntagme sur Paris : la préposition sur apparaît aussi en cooccurrence avec d'autres noms de ville. Une recherche effectuée le 20 février 2008 sur Yahoo France donne les résultats suivants pour d'autres villes de France, en cooccurrence avec le verbe être :

\begin{tabular}{|c|c|c|c|c|c|}
\hline Expression & Ville & \multicolumn{3}{|c|}{$\begin{array}{l}\grave{a} \\
\text { (Nombre d'occurrences) }\end{array}$} & $\begin{array}{l}\text { sur } \\
\text { (Nombre d'occurrences) }\end{array}$ \\
\hline \multirow{9}{*}{ Je suis } & & & & & \\
\hline & Lyon & & 17500 & 22700 & \\
\hline & Toulor & & 12100 & 15600 & \\
\hline & Marse & & 11900 & 12400 & \\
\hline & Bordea & & 4290 & 9260 & \\
\hline & Strasb & ourg & 2640 & 1440 & \\
\hline & Rouen & & 646 & 915 & \\
\hline & Orléan & & 613 & 690 & \\
\hline & Tours & & 775 & 664 & \\
\hline
\end{tabular}

77 Les noms des villes situées dans les départements et territoires d'outremer, apparaissent également, quoique en moindre proportion (entre 9 et $23 \%$ ), comme objets prépositionnels du relateur sur (Yahoo France le 30 août 2008) :

\begin{tabular}{|l|l|l|l|}
\hline Expression & Ville & $\begin{array}{l}\text { a } \\
\text { (Nombre d'occurrences) }\end{array}$ & $\begin{array}{l}\text { sur } \\
\text { (Nombre d'occurrences) }\end{array}$ \\
\hline
\end{tabular}




\begin{tabular}{|l|l|l|l|}
\hline Je suis & \multicolumn{3}{|c|}{$\mid$} \\
\cline { 2 - 4 } & Nouméa & 294 & 80 \\
\hline Papeete & 199 & 20 \\
\hline Cayenne & 96 & 19 \\
\hline Fort-de-France & 55 & 15 \\
\hline Pointe-à-Pitre & 10 & 3 \\
\hline
\end{tabular}

78 Le phénomène semble s'étendre aux noms des métropoles du monde entier avec différents degrés de saillance et un nombre variable d'occurrences sur la Toile. Ce qui se traduit par une présence plus ou moins significative de la formulation en sur. Voici les résultats de notre recherche sur Yahoo France le 23 juillet 2008 (l'ordre des villes est décroissant selon les totaux à + sur) :

\begin{tabular}{|l|l|l|l|l|}
\hline Expression & Ville & $\begin{array}{l}\text { Total } \\
\text { d'occurrences }\end{array}$ & $\begin{array}{l}\text { à } \\
\text { (Nombre } \\
\text { d'occurrences) }\end{array}$ & $\begin{array}{l}\text { sur } \\
\text { (Nombre } \\
\text { d'occurrences) }\end{array}$ \\
\hline
\end{tabular}




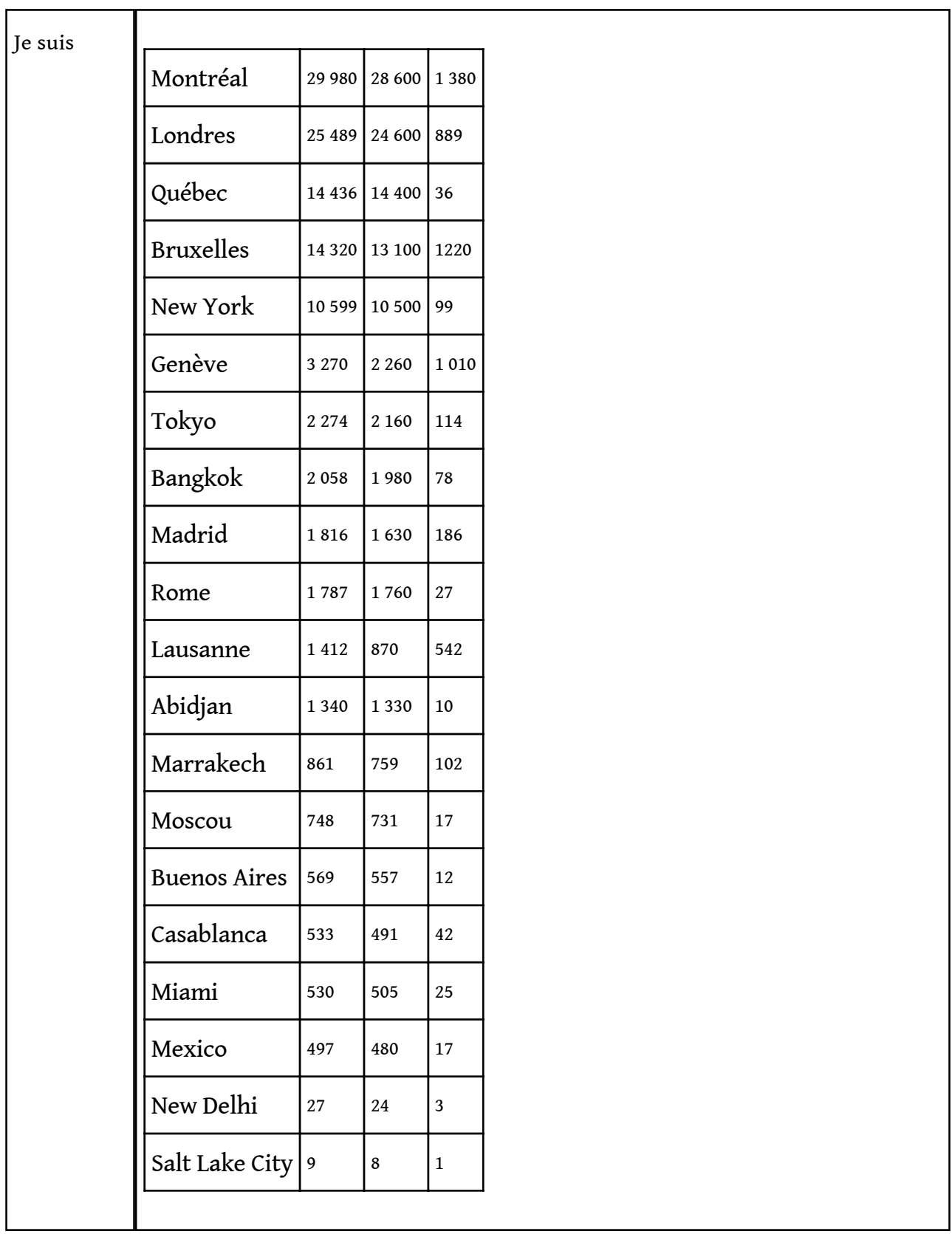

Si pour les villes situées en France le taux de cooccurrences des tournures en sur avec le verbe être oscille entre 35 et $69 \%$ (Strasbourg 35,29\%, Lyon 56,47 \%, Bordeaux 68,34\%), sur 20 villes situées ailleurs, sur n'atteint le tiers des emplois que pour Lausanne $(38,38 \%)$ et Genève (30,89 \%). Quant au reste des cas, à peine entre 10 et $12 \%$ pour Marrakech $(11,85)$, New Delhi $(11,11)$, Salt Lake City $(11,11)$ et Madrid $(10,24 \%)$; entre 7 et $9 \%$ pour Bruxelles $(8,52)$ et Casablanca $(7,88)$; entre 3 et $5,01 \%$ pour Tokyo $(5,01)$, Miami $(4,72)$, Montréal $(4,60)$, Bangkok $(3,79)$, Londres $(3,49)$ et Mexico $(3,42)$, entre 1 et $2,5 \%$ pour Moscou $(2,27)$, Buenos Aires $(2,10)$ et Rome $(1,51)$, moins de $1 \%$ pour New York $(0,93)$, Abidjan $(0,75)$ et Québec $(0,24)$.

Certes, la présence de la préposition sur devient, on le voit, beaucoup plus discrète devant le nom des villes situées hors de l'Hexagone. Pourtant, l'apparition du marqueur introduisant des toponymes urbains du monde entier prouve qu'il ne s'agit pas d'une 
tournure restreinte aux localisations domestiques mais d'un emploi en pleine expansion applicable aux agglomérations urbaines en général.

81 L'état des lieux semble donc établi :

- un sémantisme distinct pour les deux relateurs mis en regard ;

- l'émergence d'emplois concurrents ;

- un flottement, devant les noms de ville, déjà installé dans le discours avec des verbes non directionnels;

- un traitement relativement récent du phénomène qui n'apparaît comme objet d'étude que depuis une vingtaine d'années.

82 Voici venu le moment d'introduire les principes théoriques qui nous permettront de modéliser cet emploi. Dans les sections qui suivent, seront abordés les concepts de représentation et de scène verbale qui se trouveront à la base de notre dispositif d'analyse.

\section{La mise en scène de la spatialité}

\subsection{Système propositionnel et non propositionnel}

83 La linguistique cognitive développée depuis les années 80 (Jackendoff 1983, Langacker 1987) tend - explicitement - un pont entre le langage et d'autres capacités telles que la perception visuelle et le contrôle moteur, l'information mentale comprenant donc du propositionnel et $\mathrm{du}$ non propositionnel. C'est ainsi que coexistent représentations linguistiques et systèmes d'imagerie, i.e. des configurations fonctionnelles de nature « pictorielle » (Engel 1994 : 156). Décrites comme similaires aux configurations physiques, les images sont censées être analogiques et continues alors que les représentations propositionnelles sont considérées comme étant digitales, et composées d'unités discrètes. Cependant, si différents soient-ils, ces deux systèmes ne fonctionnent pas en vase clos. En effet, en présence d'un texte, par exemple, donc au départ du système propositionnel, un lecteur construit non seulement des représentations linguistiques mais aussi une représentation spatiale figurant les objets, les personnages, les événements et les relations internes de la situation décrite, dans un système non propositionnel (De Vega et Rodrigo 1997 : 51).

Similaire en principe aux modèles employés pour la perception, pour la mémoire et pour la pensée, ce type de représentation, est également à l'œuvre dans la production du discours ; c'est ce qui ressort de l'étude du processus de production de plans décrite par Christopher Habel (1997 : 104) qui, dans son cadre théorique, suit le principe proposé par S. E. Palmer ${ }^{8}$ d'une théorie générale de la représentation selon laquelle une situation de représentation est définie par l'existence d'un monde représenté (W1), d'un monde représentant (W2) et d'une relation de représentation du monde représenté au monde représentant. Mondes représentés et représentants sont définis par des systèmes de relation constitués d'un univers, c'est-à-dire un ensemble d'unités, d'un ensemble de propriétés et d'un ensemble de relations concernant cet univers. Respectant certaines de ces propriétés et de ces relations, la relation entre les deux mondes est considérée comme un homomorphisme relatif aux propriétés et aux relations choisies.

Or, on peut concevoir des mondes représentants différents par rapport à un même monde représenté : ainsi un globe terrestre et un planisphère sont des représentations de la terre. Cependant, alors que le globe terrestre respecte les relations topologiques de 
voisinage, certaines de ces relations ne sont pas explicitées dans la représentation en deux dimensions de sorte que c'est notre connaissance des conventions régissant les cartes qui nous permet de nous faire une image du monde en regardant un planisphère. Autrement dit, de même que notre monde peut être figuré par des entités dissemblables du point de vue dimensionnel, l'espace environnant peut être rendu par plus d'un système de représentation. Resterait à définir plus précisément cette relation entre représenté et représentant.

\subsection{Théorie de la représentation}

La représentation est généralement conçue comme le « renvoi d'une entité appartenant à un domaine ontologique particulier vers une autre qui relève d'un domaine différent " (Gosselin $2005: 12$ ), ainsi par exemple, mots et objets, mots et concepts, mots et images, concepts et images, etc.

Le discours de représentation (de repraesentare, "rendre présent »), qui suppose l'absence des objets et des événements évoqués, comporte un processus cognitif de dissociation des propriétés de la perception. En effet, si la perception pose des contraintes de co-présence et de coïncidence, dans la représentation, les éléments spatio-temporels de la situation perceptive, tenus pour des paramètres susceptibles de prendre différentes valeurs, seront dissociés et déplacés sur des champs de valeur (Gosselin 2005 : 25-26).

Sont ainsi distinguées perception et simulation de perception :

«La perception est ici l'acte qui place quelque chose sous les yeux comme lui-même en personne, l'acte qui constitue originairement l'objet. Le contraire est re-présentation (Vergegenwärtigung, Re-Praësentation) en tant qu'acte qui ne place pas un objet en personne sous les yeux, mais précisément le re-présente, le place pour ainsi dire en image sous les yeux, même si ce n'est pas précisément sur le mode d'une conscience d'image proprement dite. »

(E. Husserl ${ }^{9}$ (éd. 1964), pp. 57-58, que nous citons d'après Gosselin 2005 : 212)

89 Ainsi, si l'acte de représentation n'implique pas nécessairement une conscience imageante, force est de reconnaître que l'image renvoie à un objet absent à travers un contenu physique ou psychique qui ne se donne pas en propre mais comme représentant analogique de l'objet référé (Sartre 1940 : 34).

Notons que, parmi les binômes du type représentant-représenté, mots et images jouent un rôle de premier plan. Car il semblerait qu'à sa perception et sa conceptualisation du monde, l'homme ajoute une capacité verbalisante et imageante qui lui permet de le représenter par des moyens divers. Mise en mots et mise en images pourraient donc être conçues comme deux systèmes de renvoi aussi bien pour la présentation que pour la simulation de la présence d'entités ou de procès de l'univers environnant (monde réel, projeté ou imaginaire); d'où l'importance du rapport entre langage et visualisation ${ }^{10}$.

91 Dans les lignes qui suivent, le lecteur trouvera une caractérisation sommaire des dispositifs imageant et linguistique à l'œuvre dans la représentation: seront décrits successivement les propriétés des images rendant présents des objets absents ${ }^{11}$, le fonctionnement de la dissociation dans la verbalisation et les points de contact entre les deux systèmes. 


\subsection{L'image}

92 Par 'image' nous entendons ici la reproduction mentale (analogique et continue) d'une perception antérieure, voire la vision intérieure ${ }^{12}$. Cette représentation imagique diffère fondamentalement de la perception. En effet, tandis que, dans la perception, l'objet se donne par profils dans une lente construction du savoir, apparaissant sous un angle particulier selon un principe d'individuation et d'identité, dans la conscience imageante, le savoir apparaît comme immédiat car l'image livre l'objet en bloc, le faisant naître comme il est en soi, avec un coefficient de généralité et comme une synthèse de tous ses aspects (Sartre 1940 : 19-20, 119-122). Le passage de la perception à l'image suppose donc un appauvrissement de la matière et corrélativement une augmentation de la généralité :

«À mesure que la matière de la conscience imageante s'éloigne davantage de la matière de la perception, à mesure qu'elle se pénètre davantage de savoir, sa ressemblance avec l'objet de l'image s'atténue. Un phénomène nouveau apparaît: le phénomène d'équivalence. »(Sartre 1940 : 73) (C'est nous qui soulignons)

Tendant un pont entre deux domaines ontologiques, la notion d'équivalence s'avère cruciale pour le rapport représentant-représenté, pour le contact entre les différents systèmes de représentation (en l'occurrence images et mots), ainsi que, à l'intérieur d'un même système, par exemple dans le système linguistique, pour comprendre le phénomène des formulations alternatives.

94 Le rôle du savoir sera lui aussi déterminant : d'une part, pour le gommage des différences dans l'établissement de l'équivalence et, d'autre part, pour le déclenchement des inférences nécessaires au processus interprétatif.

\subsection{Le dispositif linguistique}

95 Comme il a été signalé en 2.2., dans le discours de représentation, les éléments essentiels de la perception sont conservés mais partiellement transformés, déplacés (Gosselin 2005 : 22, 26-27). Certains éléments coïncidents se trouvant disjoints par le processus de représentation, la dissociation des caractéristiques de la perception, générera des espaces ou champs de valeur sur lesquels le sujet opèrera des déplacements des entités et des procès. Cette opération de dissociation et de déplacement fera l'objet d'un balisage, effectué par les systèmes sémiotiques appropriés (système des dates, des lieux, des personnes, etc.), qui les rendra stables et publiquement repérables, donc communicables.

Ce processus libère le sujet de sa situation spatio-temporelle immédiate, rendant possibles des relations entre entités ou procès présents et absents, des faits d'interprétation et de croyance et même des mises en scène d'événements passés ou possibles. Et ce dans le cadre d'un dispositif sémantique permettant la mise en mots du montrable comme une simulation de perception. L'espace abstrait résultant du processus cognitif de représentation, devenu intersubjectif grâce à l'intervention des systèmes sémiotiques, servira de cadre à la construction de ce que Bernard Victorri $(1997,1999)$ appelle une scène verbale.

Examinons à présent le rapport entre mise en images et mise en mots. 


\subsection{Activité imageante et verbalisation}

98 Quel est le rapport établi par le sujet entre représentations linguistiques et pictorielles? S'il est vrai que la mise en mots repose sur un système issu d'une convention et que la mise en images fonctionne sur un mode analogique, il n'en reste pas moins qu'il ne s'agit pas de deux systèmes étanches. Nous l'avons dit, sans que l'on puisse établir de bijection entre les deux systèmes, les représentations mentales, modèles mentaux ou modèles de situation comprenant des figurations d'entités et de procès peuvent, soit participer à la production du discours, soit être activées par le support linguistique, par exemple lors de la lecture d'un texte. C'est cette activité imageante que visent les propos de Binet que nous transcrivons ci-après :

\footnotetext{
«Nous avons souvent des images bien plus précises que nous ne le supposons; en lisant une pièce par exemple, des images de position, de mise en scène; nous faisons sans nous en apercevoir, une plantation du décor. Il faut nous dessiner, par exemple, le plan de la scène pour qu'aussitôt nous prenions conscience de notre mise en scène personnelle, par un sentiment de résistance intérieure. »

Binet, cité par Delacroix dans le Traité de Psychologie de Dumas, t. II, p. 118, que nous citons d'après Sartre $1940: 87$. (C'est nous qui soulignons)
}

Même conception sous la plume de Jean-Paul Sartre qui considère qu' « un monde tout entier [...] apparaît en image à travers les lignes du livre» (1940:217). Le philosophe souligne le statut particulier des images générées par la lecture des textes en tant que simulation de perception :
"Lire un roman, c'est prendre une attitude générale de la conscience : cette attitude ressemble grossièrement à celle d'un spectateur, qui, au théâtre, voit le rideau se lever. Il se prépare à découvrir tout un monde, qui n'est pas celui de la perception, mais pas non plus celui des images mentales. Assister à une pièce de théâtre c'est appréhender sur les acteurs, les personnages, sur les arbres de carton la forêt de «As you like it». Lire c'est réaliser sur les signes le contact avec le monde irréel. Dans ce monde, il y a des plantes, des animaux, des campagnes, des villes, des hommes : d'abord ceux dont il est question dans le livre et puis une foule d'autres qui ne sont pas nommés mais qui sont à l'arrière-plan et qui font l'épaisseur de ce monde. (Par exemple, dans un chapitre consacré à un bal, tous les invités du bal dont on n'a rien dit mais qui sont là et qui «font nombre.») Ces êtres concrets sont les objets de mes pensées : leur existence irréelle est corrélative des synthèses que j'opère guidé par les mots. C'est que, ces synthèses même, je les opère à la façon de synthèses perceptives et non de synthèses signifiantes. » (Sartre $1940: 88$, c'est nous qui soulignons)

Le rapport existant entre activité imageante et verbalisation transparait d'ailleurs dans les manifestations langagières référant à la compréhension en termes de vision et à l'explication en termes de monstration ( « Je vois ce que vous voulez dire... »). L'association entre mise en mots et perception/monstration (Gosselin 1996: 78-86) est présente dans certaines conceptions philosophiques et psychologiques comme celle du Tractatus de Wittgenstein ${ }^{13}$ (« Nous nous faisons des tableaux des faits ») et surtout la conception de Heidegger ${ }^{14}$ : «La structure fondamentale de l'énoncé est la monstration de ce sur quoi il porte ». L'énoncé apparaît donc comme une monstration et un dévoilement ce qui fait de la communication linguistique un dévoilement partagé.

Enfin, la simulation de perception renoue avec la rhétorique. Dans Temporalité et modalité, Laurent Gosselin met en lumière les liens entre la simulation de perceptionet la rhétorique des peintures $\mathrm{du} \mathrm{XVII}^{\mathrm{e}}$ siècle où la représentation constitue non pas un simple renvoi 
sémiotique mais un processus complexe de simulation de présence cherchant à rendre présents les objets absents (2005: 19).

Associée à la communication linguistique et présente dans la rhétorique, la simulation de présence par l'image s'impose ainsi comme sujet de réflexion lançant en même temps le défi de sa propre représentation : en effet, les figurations mentales apparaîtront souvent sous les traits d'entités physiques couramment considérées comme 'images' : dessins, tableaux, photographies, photogrammes.

\subsection{Un analogon physique pour la représentation de l'imagerie}

L'appréhension de ce que l'on entend par imagerie apparaît donc comme un élément de base pour l'analyse du rapport entre représentation linguistique et figuration mentale. À ce titre, et pour satisfaire aux conditions d'une étude empirique, la mise en images est souvent visée à travers un analogon physique.

Ainsi, sont présents dans les travaux des linguistes différents équivalents correspondant à la reproduction mentale ou à la vision intérieure. Dans son analyse du présent historique, Laurent Gosselin (2005: 214) modélise la représentation - picturale ou non, présente ou absente - par analogie avec la peinture. Patrick Charaudeau (1992: 635) conçoit entre image et verbalisation, un rapport de théâtralisation : “'Communiquer', c'est procéder à une mise en scène ", affirme-t-il dans sa Grammaire du sens et de l'expression. Avec une conception théâtrale visant spécialement des effets de sens, le locuteur est assimilé à un metteur en scène qui utiliserait des ressources (l'espace scénique, les décors, la lumière, la sonorisation, les comédiens et un texte) pour produire des effets sur un public déterminé.

La métaphore théâtrale se rapproche, parfois, de la cinématographie. Le concept de scène développé par Laurent Gosselin (1996) pour une modélisation de la temporalité sur la base d'un intervalle de référence ouvert par une fenêtre de monstration suppose un défilement des événements selon le point de vue du sujet. L'auteur distingue des vues articulées en scènes un peu à la manière du montage filmique. Chez Bernard Victorri (Victorri et Fuchs 1996, Victorri 1997, 1999), l'allusion au cinéma est beaucoup plus directe: le linguiste développe la notion de mise en scène sur le modèle de l'image filmique associant les ressources langagières à la panoplie des moyens techniques au service du tournage cinématographique : choix de l'angle et du point de vue de la caméra, échelle des plans, effets de montage, etc., base sur laquelle il élabore le concept d'une scène de nature langagière appelée scène verbale.

\subsection{La scène verbale}

Dans La polysémie. Construction dynamique du sens, Bernard Victorri et Catherine Fuchs (1996: 200) concluent leurs réflexions sur le langage et la cognition par une approche de l'activité de parole en termes de construction d'espaces (Fauconnier 1984) pour développer ensuite l'idée d'un processus cognitif particulier : la construction, par le locuteur, d'un « espace extérieur à lui-même, partageable par ses interlocuteurs, et dans lequel il donne à voir ce dont il parle comme une scène projetée devant eux ». La métaphore cinématographique est justifiée et expliquée :

« [le locuteur braque] la caméra où il veut entraîner son auditoire, pouvant à sa guise suivre l'action dans son déroulement, revenir en arrière, sauter plus loin, se 
focaliser sur un détail ou au contraire prendre du champ, se mettre subitement à la place d'un de ses personnages pour montrer comment il voit les choses, comment il les a rêvées, comment il se représente à son tour l'image que s'en fait un troisième... » (Victorri et Fuchs $1996: 200$ ) Loin de se borner à l'expression du visualisable, la parole a le pouvoir de donner à voir les notions les plus abstraites. "Tout se passe comme si le langage utilisait sa capacité d'évocation de scènes concrètes pour exprimer l'ensemble du dicible» (1996: 203). En effet, la fonction de la parole, affirment les auteurs, est la construction de scènes, quel que soit l'objet du discours. Ainsi, la scène devient scène verbale, où les acteurs et leurs relations n'existent qu'en tant qu'ils sont étiquetés par des expressions linguistiques.

Comment caractériser les images évoquées par le langage? Construites dans et par le discours, les images de la scène verbale acquièrent une existence phénoménologique consistante dans la mesure où elles sont partagées par les interlocuteurs qui les appréhendent comme étant extérieures à leur conscience, étroitement associées à des formes linguistiques (1996: 206). Le statut phénoménologique de la scène deviendra plus marqué dans deux articles postérieurs où Bernard Victorri $(1997,1999)$ évoque la construction d'un espace cognitif de type particulier : un champ intersubjectif partagé par les interlocuteurs, résultat de l'activité de parole, possédant la propriété de faire surgir devant les interlocuteurs des entités, des procès, etc. que l'on ne saurait confondre ni avec les perceptions véritables ni avec les pensées non verbalisées (1999: 89) et qui ne doivent leur mode d'existence qu'à leur évocation par la parole et au sentiment partagé de pouvoir les percevoir comme résultat de cette évocation :

«L'objectif de l'énonciation est de créer, de stabiliser et d'enrichir cet espace de manière à lui donner une forme d'existence, au sens phénoménologique du terme : cet espace " apparaît » devant les interlocuteurs, il prend une consistance propre qui modifie la situation, chaque sujet devant prendre en compte ce nouvel élément qui devient à sa manière un acteur dans la relation intersubjective » (Victorri, $1997: 49$ ).

109 La scène verbale devient de ce fait une entité ayant un statut intersubjectif irréversible : pour le locuteur, mesurant la distance entre ce qu'il voulait exprimer et ses paroles, autant que pour les interlocuteurs, conscients de l'apparition de ce dit dans l'espace commun d'interlocution (1997: 49).

110 Ainsi, la notion de scène verbale s'avère apte à fournir un cadre descriptif précis mais suffisamment large pour l'analyse de différents marqueurs grammaticaux. Nous modéliserons donc l'étude de la spatialisation induite par les emplois de la préposition sur en termes cinématographiques en tant que manière de voir et de donner à voir essentiellementdynamique, avec des points de vue changeants, des perspectives diversifiées, des mouvements complexes dans une sorte de perception et de monstration, interaction entre la parole et l'image.

111 Notre thèse est que la scène construite par la mise en mots dans l'espace intersubjectif a des caractéristiques communes avec une mise en images dont le modèle communicable le plus proche serait fourni par le cinéma - "corrélat inséparable » du langage (Deleuze 1985 : 342-343) - et que ces affinités permettent de mieux décrire les phénomènes de représentation verbale objet de notre étude. 


\subsection{La mise en images}

112 Nous proposerons donc un dispositif d'analyse intégrant certains mécanismes de la technique cinématographique susceptibles de figurer notre conceptualisation de l'espace sur la base de notre perception visuelle.

La mise en scène cinématographique, comme mode de représentation de l'univers réel ou imaginaire à l'aide d'une mise en images particulière, participerait, ainsi que le langage, à la construction du sens dans un système de compositionnalité gestaltiste (Victorri et Fuchs 1996). L'émergence du sens y résulte de la participation de tous les éléments en interaction, le tout étant supérieur à la somme des parties.

114 Pourtant cette connexion entre imagerie et verbalisation ne suppose ni identité ni assimilation: le lien que nous nous proposons d'établir trouve sa raison d'être dans la présomption d'un rapport, exploitable à des fins heuristiques, entre deux systèmes de représentation sur la base de l'affinité entre certaines caractéristiques de l'image filmique et les mécanismes de la perception visuelle.

115 Pour la mise en forme de notre dispositif d'analyse, nous adapterons certains concepts de la technique cinématographique.

\subsection{Phénomènes propres à la mise en images cinématographique}

116 Le langage cinématographique fournit un large éventail de dispositifs d'analyse susceptibles d'intégrer une étude linguistique : focalisations changeantes, profondeur de champ, rapports entre images multiples, ellipses, consécutions et simultanéités, etc. Parmi les éléments de cette panoplie, nous nous intéresserons ici aux phénomènes de cadrage. Et ce à double titre : en tant que phénomène de partition, par la circonscription d'un champ, et en tant que phénomène de sélection, par l'inclusion/exclusion d'éléments dans un espace borné, ce double aspect se trouvant d'ailleurs à la base des réflexions sur l'organisation de la perception.

117 D'abord, le découpage d'une portion du montrable. En termes cinématographiques, nous dirons que le sujet définit un champ d'observation dans lequel il opère un cadrage semblable à celui d'une caméra, qui correspond à son angle de vue ou à celui de son œil mental (Talmy 1983). Ce cadrage détermine un champ, fragment de l'espace retenu dans l'image, selon un cadre, limite exacte de ce champ dans un jeu serré entre espaces in et off . Au champ comprenant ce qui est présent dans l'image s'oppose le hors champ, c'est-àdire, ce qui n'est pas présent sur l'image. Du point de vue technique, dans le cadre, limite exacte du champ, la caméra retient un plan (une série de photogrammes successifs enregistrés entre la mise en marche et l'arrêt de l'appareil) que nous prendrons comme unité minimale. Une succession de plans déterminera une scène (unité dramatique) ou une séquence (unité narrative).

118 Le tracé d'une frontière in / off s'accompagne, selon le périmètre de l'espace découpé et la distribution de l'attention, d'une opération de sélection des entités qui peupleront ce champ. À l'instar de l'œil qui embrasse un ensemble ou focalise un détail, le cadre s'organise selon une échelle de plans, à savoir :

- le plan général qui embrasse un paysage ou un vaste décor dans lesquels les personnages ne sont pas reconnaissables; 
- le plan d'ensemble qui montre un groupe de personnages reconnaissables dans un espace large ;

- le plan moyen qui cadre les personnages en pied. Le décor y joue un rôle moins important, ce sont les gestes et les mouvements des personnages qui se trouvent mis en valeur ;

- le plan américain qui cadre les personnages à mi-cuisse concentrant l'attention sur le buste et les bras ;

- le plan demi-rapproché qui cadre les personnages à la taille ;

- le plan rapproché qui cadre les personnages à la poitrine ;

- le gros plan qui cadre le visage ;

- le très gros plan qui présente un détail du visage ou du corps d'un personnage (les yeux, la main...);

- l'insert ou gros plan d'objet.

Pourquoi nous intéresser au cadrage déterminant un champ selon une échelle de plans? Surtout en vertu de l'omniprésence de cette opération qui traverse les réflexions sur la perception, la cognition, le langage. En tant que délimitation d'un ensemble, le cadrage se trouve en outre intégré à la modélisation de certaines manifestations langagières relatives au champ visuel, au champ attentionnel et à la construction du sens.

En effet, les faits de cadrage, base de notre modélisation se trouvent présents dans l'opération de fenêtrage (Talmy, 2000 ; Col et Victorri, 2007). Leonard Talmy, qui distingue sa théorie de la théorie des «cadres" (frames) de Fillmore, lie explicitement le windowing of attention, en tant qu'opération conceptuelle générique, à la structuration d'autres systèmes cognitifs comme la perception visuelle. Le fenêtrage d'attention se caractérise par l'inclusion ou l'omission des éléments d'une scène, sur la base du processus cognitif de distribution sélective de l'attention. Dans le discours, cette opération se manifeste par l'inclusion du matériel linguistique explicite, pour les portions fenêtrées, et l'exclusion, pour les portions omises.

Cette sélection, qui a des points communs avec les phénomènes de cadrage cinématographique que nous proposons, suppose, de manière inhérente :

- la détermination, par une frontière (plus ou moins précise), d'une région de cohérence, de co-importance et de connectivité (2000 : 305). Autrement dit, selon notre point de vue, la fenêtre semble cadrer un champ ;

- une dichotomie fenêtrage - omission (windowing - gapping) générant une disjonction entre l'ensemble montré et ce qui se trouve omis. Notre dispositif rendra opératoire cette disjonction sous la forme d'une tension entre champ et hors-champ qui adoptera deux formes fondamentales : hors-champ relatif et hors-champ absolu (Deleuze 1983) ;

- le déclenchement d'une inférence des éléments non fenêtrés, dans un contexte donné, sur la base du savoir ;

- la possibilité de cadrages alternatifs émergeant de la flexibilité caractéristique du système attentionnel. C'est sur cette base qu'une même scène peut avoir un fenêtrage initial, médian ou final (Talmy 2000 : 266). Dans notre analyse, il s'agira d'une échelle de plans variable.

Le fenêtrage, en tant que modélisation, apparaît aussi dans les récents développements de Gilles Col et Bernard Victorri (2007) qui établissent explicitement le lien entre fenêtrage et représentation en tant que déplacement d'attention (Gosselin, 2005). Les auteurs, qui intègrent la dimension temporelle à la fenêtre d'attention, trouvent dans l'opération de fenêtrage une formalisation compatible avec la définition de scène verbale et apte à rendre compte de la construction du sens temporel (en l'occurrence l'évocation de l'avenir en anglais) du point de vue énonciatif et cognitif. 
Il apparaît ainsi que, en tant que délimitation d'espaces du point de vue cognitif et langagier, les opérations de cadrage (comme monstration à travers un champ délimité, appelé fenêtre ou autre) jouent un rôle de premier plan dans l'étude de la construction du sens.

Cette présentation sommaire des phénomènes de cadrage sera complétée par une révision, en termes de dispositif cinématographique, de deux études linguistiques (Gosselin 1996 et Talmy 1983) ce qui nous permettra, d'abord, de trouver des points communs entre fenêtrage et mise en images, ensuite, de définir une unité minimale d'analyse et, enfin, de tester la pertinence de notre dispositif. Nous définirons ainsi le concept de plan comme unité minimale et testerons l'existence d'une échelle de plans dans la production/interprétation d'énoncés.

\subsubsection{Distinction entre vue et plan d'après un exemple de Laurent Gosselin (1996)}

Sur la base d'une spatialisation du temps sous forme de deux trajectoires d'orientation inverse représentant la temporalité du sujet et celle de l'objet, Laurent Gosselin (1996 : 81) élabore une Gestalt conceptuelle qui représente la perception/monstration du temps dans l'énoncé. Décrite métaphoriquement en termes spatiaux, la perception définit un champ à l'intérieur duquel défilent les changements survenant au rythme du déroulement temporel.

Appliqué à la narrativité, ce schéma, figurant la temporalité comme une double flèche du temps, illustre la manière dont les événements se succèdent selon la dynamique temporelle de l'objet à mesure que le récit avance sur l'axe du sujet. Le dispositif d'analyse se laisse décrire à partir des notions suivantes (1996: 96) :

- un intervalle de référence, construit pour les besoins d'analyse, sur l'axe temporel, correspondant à ce qui est perçu ou montré au moment où l'énonciateur décide de parler ;

- une fenêtre ouverte, par le sujet, sur une scène ;

- la scène, entité perceptive constituée de l'ensemble des procès qui défilent dans une même fenêtre ;

- l'espace occupé para la scène ;

- le point de vue du sujet ;

- une vue ou, en général, une succession de vues composant la scène, la vue étant ce qui est perçu/montré à un même moment du temps. Son unité est fondée sur l'identité de ce qui est perçu/montré à travers la fenêtre : un changement dans le champ perceptif entraîne un changement de vue. Par exemple, la scène comprenant trois vues (V) successives :

$\mathrm{V} 1$ : Luc dormait. Les volets étaient clos.

V 2 : Il se réveilla quand Marie entra. Elle marchait pourtant sur la pointe des pieds. V 3 : Il ouvrit les volets.

Adapté aux besoins de l'étude de la temporalité, l'exemple des trois vues présenté cidessus établit trois moments. Luc dormait et Les volets étaient clos correspondent à un seul moment et peuvent être représentés comme concomitants avec une hiérarchisation possible entre action principale (figure) et arrière-plan (fond). Il en va de même pour la deuxième vue : Il se réveilla quand Marie entra, correspondant au même moment que Elle marchait pourtant sur la pointe des pieds, est susceptible d'une organisation hiérarchique interne. Quant à la troisième vue (Il ouvrit les volets), elle est irréductible à une telle division car elle correspond à un seul plan. 

successifs.

V 1 :

V 2:

V 3: différente : ville. (1983)

Or, ce même exemple pourrait être analysé différemment en vue d'une étude de la spatialité. En effet, l'introduction d'une unité spécifique pour le cadrage de la scène, par exemple le plan, - portion du champ d'observation compris dans les limites d'un cadrage particulier et assimilable, sous sa forme verbale, à un énoncé - permettrait de sélectionner, pour une même unité de lieu (la chambre de Luc) plusieurs cadrages

Ainsi, dans l'exemple étudié

Plan $1:$ Luc dormait.

Plan 2 : Les volets étaient clos.

Plan 3 : Il se réveilla quand Marie entra.

Plan 4 : Elle marchait pourtant sur la pointe des pieds.

Plan 5 : Il ouvrit les volets.

Chacun des plans établis, est caractérisé par une « grosseur » différente : ainsi les plans 1 , 2 et 5 pourraient être conçus comme des plans moyens ou demi-rapprochés, alors que le plan 3 doit être assez large pour cadrer les deux personnages en même temps (plan d'ensemble) et que le plan 4, plan moyen permettant de voir Marie en pied, pourrait faire l'objet d'un effet de zoom sur les pieds du personnage.

La première vue serait donc constituée de deux plans :

Luc dormait. Les volets étaient clos.

Plan $1 \quad$ Plan 2

avec le montage de deux plans moyens ou rapprochés cadrant d'abord Luc, ensuite la fenêtre aux volets clos.

Comparons avec un seul plan général :

Luc dormait dans la pénombre d'une chambre aux volets clos

où sont perçus/montrés Luc, la chambre et les volets. Ou avec deux plans de grosseur

Les volets clos, la chambre baignait dans une douce pénombre. Luc dormait où un plan général de la chambre est suivi d'un plan moyen ou rapproché du personnage.

Il résulte de ce qui précède que, à la structuration de la scène en vues dans une approche temporelle et processuelle, il semble possible d'ajouter, avec une conception visuelle de l'espace perçu et montré, un découpage en plans en tant qu'unités perceptives déterminées par un cadrage particulier permettant des alternatives de mise en scène aussi bien visuelle que linguistique. La notion de cadrage sera mise à contribution pour l'appréhension de la tension entre champ et hors-champ que nous développerons en 2.10. Cette dialectique sera cruciale pour l'analyse de la concurrence $\grave{a}-$ sur devant les noms de

\subsubsection{Mise en images et échelle de plans selon un exemple de Leonard Talmy}

7 Dans sa description des quatre systèmes d'imagerie correspondant au niveau de structuration grammatical et syntaxique, Leonard Talmy (1983), sans allusion explicite au cinéma, a recours à des éléments de visualisation cinématographique. Par exemple, le 
troisième système (1983 : 256) qui spécifie la distribution de l'attention accordée à une scène, depuis un point de vue indiqué, est illustré comme suit :

(a) Une focalisation sur les détails les plus fins :

There are freckles on the boy's face

(Il y a des taches de rousseur sur le visage de l'enfant)

(b) une focalisation d'une portée moyenne :

The boy's face has freckles on it

(Le visage de l'enfant a des taches de rousseur)

(c) une focalisation sur le cadre général

The boy has freckles on his face

(L'enfant a des taches de rousseur sur le visage)

Ces trois exemples illustrent une focalisation décroissante des taches de rousseur. Or ce système d'imagerie, jouant en principe sur la dialectique figure-fond selon un phénomène d'attention, semble pourtant lié à l'échelle des plans. En effet, les énoncés

Il y a des taches de rousseur sur le visage de l'enfant

Le visage de l'enfant a des taches de rousseur

L'enfant a des taches de rousseur sur le visage

pourraient correspondre à une échelle de plans croissante : un très gros plan des taches de rousseur, un gros plan sur le visage et un plan demi rapproché ou moyen de l'enfant.

Des systèmes d'imagerie proposés par le linguiste américain, nous retiendrons non seulement le rapport entre image et verbalisation mais aussi le concept de cadrages alternatifs pour une même scène, dans l'exemple étudié, selon la variation dans l'échelle des plans. Dans notre analyse de l'alternance prépositionnelle entre à et sur, nous traiterons la sélection prépositionnelle comme une mise en images alternative.

\subsection{3. Éléments de modélisation}

Ce bref parcours nous a permis d'établir la pertinence du lien entre verbalisation et imagerie, lien qui sous-tend des modélisations telles que la scène verbale et l'opération de fenêtrage. En effet, les limites du viseur de la caméra lors de la prise de vues et le contour de l'écran de projection modélisent la perception/monstration à travers des cadres assimilables aux fenêtres qui fractionnent un espace d'observation et sélectionnent les entités/procès évoqués par le discours.

L'importance du cadrage ayant ainsi été mise au jour, notre modélisation prendra comme base deux notions :

- la dimension du plan (assimilable à un énoncé) découpé par le cadrage déterminera l'ensemble des unités perçues/montrées ;

- la délimitation du cadre générera une tension entre champ et hors-champ i.e. vu (montré) et non vu (non montré), susceptible de déclencher des inférences selon les contextes.

Cette dernière notion, essentielle aux opérations imageantes autant qu'aux opérations de fenêtrage, sera intégrée à notre dispositif d'analyse de la mise en mots de la spatialité comme manière de voir et de donner à voir l'univers environnant.

\subsection{Le hors-champ}

Qu'est-ce que le hors-champ? Du point de vue technique, se trouve hors champ tout ce qui n'est pas dans le champ de vision, ce dernier comprenant ce qui est présent dans l'image 
comme résultat d'un certain cadrage. Autrement dit, le hors-champ résulte inévitablement d'un cadrage: qu'il soit conçu comme un enfermement de toutes ses composantes ou comme un prélèvement sur une aire de l'espace ou de l'action, tout cadre détermine un hors-champ.

Pour Gilles Deleuze, le hors-champ n'est pas une négation : "[il] renvoie à ce qu'on n'entend ni ne voit, pourtant parfaitement présent " (1983: 28, c'est nous qui soulignons). Car tout ensemble se prolonge dans un ensemble plus vaste avec lequel il communique. Mais, signale le philosophe, il existe, dans le hors-champ, deux aspects qui diffèrent par nature $(1983: 30)$ :

- un hors-champ relatif, de nature spatiale ;

- un hors-champ absolu, à dimension temporelle et spirituelle.

Cette distinction se trouve liée, dans la philosophie de Deleuze, à la distinction bergsonienne entre l'ensemble et le tout $(1983: 20)$.

\subsubsection{Le hors-champ relatif}

Pour caractériser le hors-champ relatif, nous redéfinirons le cadrage à la manière deleuzienne : le cadrage, déterminant un champ de vision, suppose le choix des parties qui entrent dans un ensemble, les ensembles étant par définition toujours clos. Selon Deleuze, un ensemble étant cadré, donc vu, il existera toujours un plus grand ensemble avec lequel le premier en forme un plus grand, et qui peut être vu à son tour, suscitant de la sorte un nouveau hors-champ et ainsi de suite, comme dans une mise en abyme infinie. De sorte que le hors-champ relatif, qui apparait tout autour de l'ensemble cadré, se trouverait dans le champ d'un ensemble qui l'englobe. C'est-à-dire que tout fragment de l'espace sélectionné par l'observateur serait susceptible d'être prolongé indéfiniment dans un champ plus vaste.

151 Ce qui pourrait trouver une application, par exemple, dans les formes de nomination et de catégorisation : ainsi, cadrer l'ensemble des voitures haut de gamme laisse supposer, en hors-champ relatif, l'existence de voitures de moyenne gamme ou bas de gamme, qui peuvent, à leur tour, être situées dans l'ensemble des voitures lequel se situe à son tour dans l'ensemble des véhicules. Un autre exemple, dans le domaine de la spatialité: les "localisations emboîtées" (Vandeloise 1988: 129) où l'emploi de l'indéfini avec la préposition à devient plus acceptable lorsque le site est intégré dans un ensemble plus grand :

? Elizabeth est à un guichet

Elizabeth est à un guichet à la poste

Le hors-champ relatif sera donc toujours en rapport avec un ensemble plus vaste dans lequel il se trouve inséré. Cependant tout ensemble, si vaste soit-il, demeure toujours un ensemble situé dans l'espace; c'est pourquoi il ne saurait être confondu avec le tout, à dimension temporelle et spirituelle, étroitement lié à la notion de hors-champ absolu.

\subsubsection{Le hors-champ absolu}

153 Si l'ensemble se présente comme étant constitué de parties et toujours clos, le tout, selon G. Deleuze, est ouvert et, n'ayant pas de parties, pourrait être conçu comme une continuité indivisible. «Le tout se crée et ne cesse de se créer dans une autre dimension, sans parties» (1983: 21). Pour le philosophe, les ensembles sont dans l'espace, et le tout, 
les touts, sont dans la durée même en tant qu'elle ne cesse pas de changer. En même temps, comme pur devenir sans arrêt, le tout est spirituel ou mental. «Dans un cas, dit-il, le hors-champ désigne ce qui existe ailleurs, à côté ou autour ; dans l'autre cas, le horschamp témoigne d'une présence plus inquiétante [...] un Ailleurs plus radical, hors de l'espace et du temps homogènes. Sans doute ces deux aspects du hors-champ se mélangent constamment ».

Ainsi, affirme le philosophe, alors que le hors-champ relatif ajoute de l'espace à l'espace (en prolongeant les ensembles dans une mise en abyme) dans un rapport actualisable avec d'autres ensembles, le hors-champ absolu introduit du spirituel, un rapport avec le tout qui n'est jamais parfaitement clos, dans un jeu de relations purement pensées.

C'est cette dernière notion qui nous permettra de modéliser les inférences déclenchées par les emplois prépositionnels étudiés (Cf. 3.2.).

\section{Une modélisation de la séquence sur+ville en termes de hors-champ absolu}

La première partie de ce travail a été consacrée à l'étude de l'alternance entre à et sur devant les noms de ville. Un bref portrait sémantique des deux marqueurs, sur la base d'une distinction entre localisation et configuration, a été suivi de l'identification de certains cas de flottement en discours, notamment devant des toponymes urbains. Une recherche sur un corpus électronique récent nous a permis de mesurer l'essor de ce type d'emploi prépositionnel avec des verbes situatifs.

Dans la deuxième partie de notre étude, nous avons procédé à l'élaboration d'un dispositif qui, inspiré de la scène verbale, fait écho à d'autres opérations de cadrage développées récemment. Sur la base d'une mise en images du montrable, notre modélisation - de manière informelle pour l'instant - avance particulièrement dans le traitement de la tension entre $v u /$ non $v u$ dans la perception / monstration des entités et procès objet du discours.

Le troisième volet qui s'ouvre ici sera l'occasion d'une synthèse de nos réflexions et d'une mise à l'épreuve de notre dispositif : le concept de hors champ absolu sera appliqué au traitement de l'émergence de la formulation sur+ville.

\subsection{L'environnement linguistique où se produisent les commutations entre à et sur}

Pour mémoire, en mars 2006 et mars 2008, nous avons cherché sur Yahoo France la récurrence des énoncés situatifs du type Je suis sur Paris. Le résultat a mis en évidence l'expansion de cet emploi prépositionnel qui concurrence sévèrement les constructions avec $\grave{a}$, notamment avec le verbe être : 56000 occurrences du syntagme sur Paris contre 38 000 de la séquence à Paris en 2006, 111000 contre 170000 en 2008. Ce qui consacre cette formulation comme tendance durable dans les habitudes discursives.

Pour mieux saisir les particularités de l'emploi situatif sur Paris, examinons le co-texte immédiat où se produit l'alternance prépositionnelle. 
161 La lecture des trente premières occurrences des énoncés comportant l'expression sur Paris relevés en 2006 et 2008 met en lumière la cooccurrence récurrente d'éléments disjonctifs. D'abord, un bornage temporel avec les verbes être, travailler, rester, dormir.

Ainsi, pour Je suis sur Paris :

depuis quelques années /six ans /sept mois

cela ne fait pas longtemps que...

ça fait un mois /deux ans

pour faire un stage de trois mois / six mois

toute la semaine jusqu'au vendredi non compris

pendant la semaine qui arrive et en vacances

si c'est au mois de décembre

moi perso je viens de la campagne et je suis sur Paris depuis six ans

(Yahoo France mars 2006)

Ok pour moi, je suis sur paris de temps en temps à l'hôtel ${ }^{15}$

un petit billet pour te dire que je suis sur Paris en début de semaine et que j'ai...

Je suis sur Paris la semaine prochaine de Lundi à Jeudi donc ça serait bien de...

je suis sur Paris environ 1 semaine par mois pour mon travail

je suis sur paris depuis 2 semaines je viens de montpellier d'origine

cela fait 5 mois que je suis sur paris et je n'arrive toujours pas à...

ça fait pas longtemps que je suis sur paris pour mes études...

(Yahoo France mai 2008)

Je suis sur Paris en ce moment et je viens de rayer un de mes DVD dans le portable.

Quelqu'un a une bonne adresse d'un magasin qui ferait...

Je m'appelle Sonia et je viens de Moscowa et je suis sur Paris maintenant. Je ne connais pas du monde pour l'instant ici et j'aimerais...

Voila je suis de province et le week end de 7 juillet je suis sur paris avec des amis et nous souhaiterions aller manger dans un endroit dépaysant sympa mais pas très cher...

je suis sur paris pendant qlqs jours et j'aimerai connaitre les meilleurs boutiques de BD occasions dans notre tres chere capitale...

(Google France mai 2008)

La formulation Je reste sur Paris se trouve, elle aussi, associée à des précisions temporelles : six mois par an, l'année prochaine, cet été, environ deux semaines, pour l'instant, maintenant, normalement, je ne sais pas encore jusqu'à quand, souvent accompagnées de l'expression de

\section{l'exception :}

Si je fais le pont mais je reste sur Paris il faut que je bosse sur mon mémoire

Une fois n'est pas coutume, je reste sur Paris ce soir

Moi je descends pas, je reste sur Paris tout juillet

(Yahoo France mars 2006)

L'énoncé Je dors sur Paris semble lui aussi en rapport avec un événement exceptionnel ou l'étape d'un déplacement avec des indications temporelles du type : Mardi, j'arrive à six heures et je dors sur Paris (Yahoo France mars 2006).

Aux précisions temporelles limitatives du séjour - dimension « temporaire » (Franckel et Paillard 2007 : 95) - s'ajoute une disjonction de la relation spatiale, souvent sous la forme d'un contraste entre deux espaces concurrents occupés alternativement par le sujet qui se trouve donc partagé, ce qui renforce d'ailleurs la discontinuité du rapport. Ainsi, Je travaille sur Paris, apparait en contraste avec le verbe habiter (facultativement vivre ou être) :

$\mathrm{J}$ 'habite en province/en région/la banlieue mais je travaille sur Paris,

Je travaille sur Paris et j'habite assez loin/à l'Essonne, je vis à Enghien, je suis sur

Arpajon 
Je travaille sur Paris mais je rentre souvent sur la région de Vichy

(Yahoo France mars 2006)

je suis sur paris \& champigny sur marne

salut marseille, alors maintenant je suis sur paris mais je souhaite m'installer...

Je suis sur Paris (enfin je bosse à Paris, dans le XVeme, mais je vis dans le $92 . .$.

(Yahoo France mai 2008)

bonjour, je suis sur paris mais je descend sur bagnols en forêt en septembre

Par defaut moi je suis sur Paris. J'arrive pas a me mettre sur toulouse (c'est la que

j'habite en faite pas a Paris...)

je suis en déplacement 3 jours sur 7 et quand je suis sur Paris je...

L'avantage du système que je pratique est la possibilité régulière de rencontrer des collègues de travail (lorsque je suis sur Paris) et...

(Google France mai 2008)

Il faudrait souligner en outre le manque d'emplois du relateur sur dans des co-textes induisant la coïncidence, la fixation, l'appartenance. Ces notions, selon notre corpus, émergent d'énoncés avec la préposition à, qui, elle, cumule les deux emplois, fixe et mobile :

Je suis à Paris, tellement à Paris, regardant les coins de rue comme des...

Je me promène tout autour, dans des ruelles charmantes et ensorcelantes qui me tournent la tête, je suis à Paris

Je suis à Paris, j'y vis, j'y dors et j'y travaille...

Non, je me sens américain quand je suis ici et français lorsque je suis à Paris. J'ai

véritablement un pied dans chaque pays ${ }^{16}$.

(Google France mai 2008)

166 Une recherche de possibles contre-exemples sur Yahoo France semble confirmer notre intuition. D'abord, il faut noter le manque de cooccurrence de sur Paris avec des termes dénotant une localisation stable. Et ce, aussi bien en 2006 qu'en 2008. Seule exception, l'avancée de sur Paris avec « toujours », combinaison qui progresse de 0 à 11,79\%.

\begin{tabular}{|l|l|l|l|l|l|}
\hline \multirow{2}{*}{$\begin{array}{l}\text { Expressions } \\
\text { cooccurrentes }\end{array}$} & \multicolumn{3}{|l|}{ Septembre 2006 } & \multicolumn{2}{|l|}{ Mai 2008 } \\
\cline { 2 - 5 } & $\begin{array}{l}\text { Nombre } \\
\text { d'occurrences }\end{array}$ & \multicolumn{2}{|l|}{$\begin{array}{l}\text { Nombre } \\
\text { d'occurrences }\end{array}$} \\
\cline { 2 - 6 } & \multicolumn{2}{|l|}{ à Paris } & sur Paris & à Paris & sur Paris \\
\hline J'ai toujours vécu & 76 & 0 & 317 & 29 \\
\hline J'ai passé toute ma vie & 2 & 0 & 16 & 0 \\
\hline Je me suis installé & 2 & 0 & 3 & 0 \\
\hline définitivement & & & & \\
\hline J'ai élu domicile & 1 & 0 & 1 & 0 \\
\hline
\end{tabular}

167 En outre, et sans que cela ait un caractère définitoire, certaines formulations relatives au commencement de l'existence et à la cessation de la vie, n'acceptent que très rarement la préposition sur: 


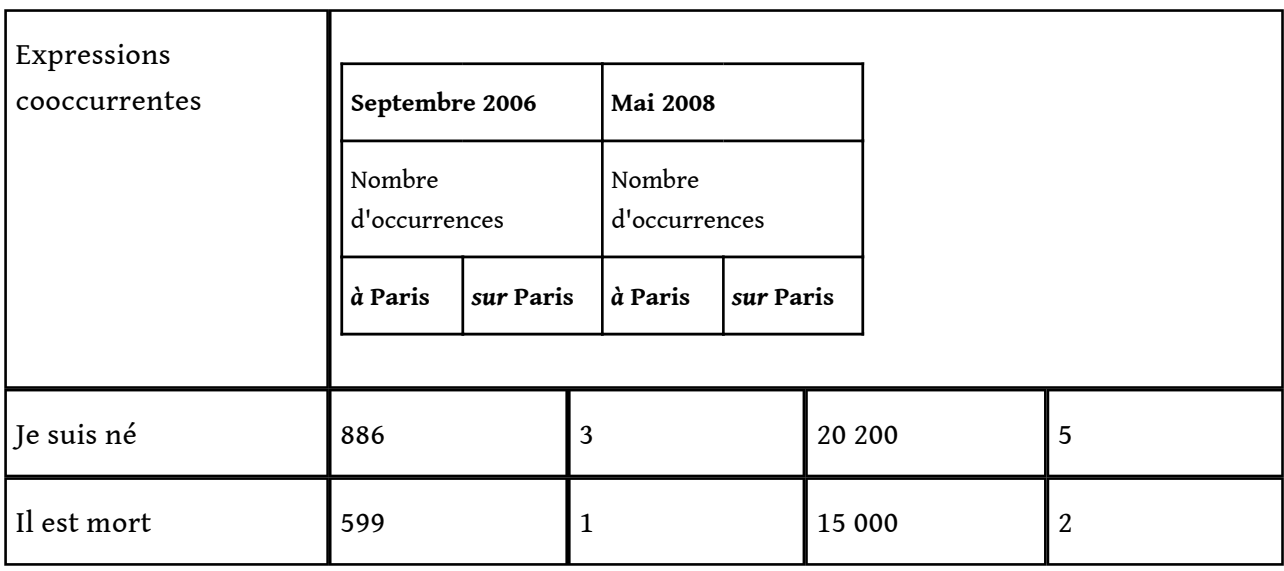

Remarquons que les occurrences de Je suis né sur Paris sont souvent suivies d'une expression de contradiction manifestée par un manque d'adhésion aux valeurs/habitudes du lieu de naissance - le sujet qualifié par le procès ne se sentant pas « parisien », comme le signale Denis Paillard (Franckel et Paillard 2007) - ou par un départ précipité :

Je suis né sur Paris mais je suis totalement pour l'OM...

Je suis né sur Paris mais je crois que je ne m'habituerais jamais...

Je suis né sur Paris (et j'y habite) mais deux jours après ma naissance, j'étais en Bretagne! Car...

Ce dernier énoncé qui semblerait contenir autant un contre-exemple (et j'y habite) qu'une nuance de contingence (deux jours après ma naissance j'étais en Bretagne) met en évidence la nuance de discontinuité présente dans les formulations en sur.

À noter que la localisation induite par la préposition à peut être, ainsi que le signale Littré, définitive ou provisoire, comme l'illustre l'énoncé

Je suis né à Paris mais je suis vite parti

Autrement dit, alors que à peut indiquer autant la localisation stable que la situation provisoire, la préposition sur semble induire un séjour discontinu, quelle qu'en soit la durée.

Quant à la seule cooccurrence de sur Paris avec le verbe mourir, elle apparaît comme étant peu représentative car elle ne réfère pas à la ville de Paris proprement dite mais au point de départ d'un trajet (Paris-Nice) :

alors le titre de ton topic n'est pas bon puisqu'il est mort sur Paris-Nice masi bon après tout on s'en fout

Une deuxième apparition de ce même énoncé sous forme de citation portera le nombre d'occurrences à 2 en 2008.

Ajoutons à ces exemples le contraste entre "X est enterré à Paris » avec 506 occurrences et « X est enterré sur Paris » qui n'enregistre aucun emploi.

Il semblerait donc que les énoncés en sur convoquent des marqueurs de contingence (statut provisoire ou mobile de la localisation spatio-temporelle), refusant les indications de stabilité spatio-temporelle, voire, notionnelle.

Bref, que ce soit par bornage temporel, par exception, par disjonction spatiale ou même par disjonction notionnelle, le caractère situatif des énoncés en sur semble généralement lié à une situation relativement récente ou susceptible de changer 
prochainement ou soumise à une variation habituelle, en tout cas provisoire plutôt que définitivement établie; autrement dit, les énoncés en sur seraient empreints d'une certaine mobilité.

\subsection{Interprétation de l'opposition à + ville vs sur +ville en termes de hors-champ absolu}

Comme nous l'avons vu, à la différence de je suis à Paris indiquant une simple localisation, je suis sur Paris suggère un bornage temporel qui, par le marquage du début ou de la fin de la relation spatiale, instaure une situation considérée comme provisoire, parfois exceptionnelle. Dans le cas du verbe rester qui possède, lui aussi, un caractère situatif (continuer à être dans un lieu), l'emploi de sur peut mettre en relief la potentialité d'un départ contribuant à accentuer la nature temporaire du séjour envisagé. Il en va de même pour le verbe dormir (être dans un état de sommeil) : je dors sur Paris semble faire allusion non pas à une habitude ou à un lieu fixe mais à une localisation discontinue. Dans le cas de la disjonction spatiale, par exemple dans travailler sur Paris, l'énoncé contribue à l'évocation d'une localisation transitoire et rend présent un ailleurs en concurrence avec le site évoqué.

178 Analysées en termes de mise en images, de telles formulations s'avèrent particulièrement intéressantes. La relation spatiale induite par la préposition sur semble accorder une importance partagée à la fixation et à son contraire : si être à Paris induit une localisation établie, être sur Paris localise tout en permettant de concevoir le fait de, tôt ou tard, ne pas être à Paris ou être ailleurs.

L'alternance prépositionnelle à / sur devant les noms de ville, se laisserait ainsi décrire comme une mise en images alternative. En effet, dans une opération de cadrage de la scène, alors que, dans je suis / reste / dors / travaille à Paris, l'entité à situer, le lieu de référence et la relation de localisation sont rendus par un plan général à l'intérieur du cadre établi par l'énoncé, dans je suis / reste / dors / travaille sur Paris, à la localisation cadrée s'ajoute le hors-champ désignant l'ailleurs (je ne suis pas toujours à Paris, je n'y suis que pour peu de temps, je n'habite pas Paris, je ne suis pas parisien, etc.)qui n' étant pas le véritable objet du dire, n'est pas directement perçu — c'est le non-vu (Deleuze $1983: 28-31$ ) -, mais parfaitement présent dans un jeu de relations pensées.

En effet, le bornage temporel, l'exception et la disjonction spatiale ou notionnelle imprégnant d'instabilité la situation de coïncidence spatiale et induisant une localisation provisoire, aident à construire un repérage non définitif empreint de mobilité, d'autonomie et de réactivité, notions valorisées à l'heure actuelle et présentes dans le discours quotidien. Aussi, par la possibilité de localiser tout en marquant la disjonction, la préposition sur contribue-t-elle à la création d'un positionnement composite, fixe et mobile en même temps qui semble mettre en jeu, à égalité, vu (présence) et non-vu (non présence), autrement dit champ et hors-champ.

181 Ce non-vu / non-montré, c'est-à-dire le début récent ou la fin imminente de la coïncidence spatiale, une localisation habituelle dans un ailleurs, une non-appartenance notionnelle, semble avoir les caractéristiques non pas d'un simple hors-champ relatif, élargissant spatialement un ensemble borné (comme dans le cas des localisations emboîtées), mais plutôt celles d'un hors-champ absolu, renvoyant à tout ce qui est de l'ordre du virtuel, tout ce qui pourrait ou aurait pu être. Ce hors-champ absolu dépasse 
donc le cadre de la localisation spatiale et, intégrant le temporel et le notionnel, apparait étroitement lié aux processus interprétatifs.

\subsection{Le hors-champ comme mode de l'implicite et la préposition comme déclencheur d'inférence}

Renvoyant à un non-vu parfaitement présent aussi bien dans la mise en images que dans la mise en mots, le hors-champémerge donc dans le discours comme mode de l'implicite. Activé par le choix d'un marqueur prépositionnel (sur), donc inscrit dans l'énoncé, il se distingue du sous-entendu (correspondant à un implicite pragmatique) et peut être apparenté au présupposé ainsi qu'à la notion de préconstruit. Quelles sont les convergences et les divergences entre le hors-champ et ces deux autres notions?

Le présupposé (comme non posé) est généralement vu comme ce qui n'étant pas posé constitue cependant une inférence nécessaire, inscrite dans la structure linguistique de l'énoncé et donc relativement indépendante de la situation de discours. Certains exemples sont marquants, tel le cas du verbe cesser, par exemple dans Jacques a cessé de fumer, qui implique nécessairement Jacques fumait (Ducrot 1984) car, évidemment, ne cesse que ce qui se produit.

Cette notion s'applique-t-elle aux formulations du type Je suis sur Paris? On peut signaler certes des points communs avec le présupposé en ce sens qu'il existe une inscription dans l'énoncé. Pourtant, si le marqueur prépositionnel est susceptible de déclencher une inférence d'instabilité, celle-ci diffère des inférences nécessaires à l'œuvre dans la présupposition. En effet, si Paul a cessé de fumer suppose bel et bien Paul fumait, Paul est sur Paris ne comporte pas Paul va quitter Paris comme inférence nécessaire. La formulation en sur introduit une composante d'instabilité par rapport à l'emploi du relateur à mais en y renvoyant à une multiplicité de possibles : Paul va quitter Paris, mais aussi Paul était ailleurs, Paul travaille / habite ailleurs, Paul ne se sent pas forcément appartenir à la ville où il se trouve, Paul n'est pas habituellement là, etc. Autrement dit, le hors-champ semble renvoyer à une glose et non pas à un contenu propositionnel précis.

Conçu par M. Pêcheux, à la suite de P. Henry, comme manifestation d'assujettissement idéologique, le préconstruit, en tant que trace dans l'énoncé d'un discours antérieur, extérieur, en tout cas indépendant (Pêcheux 1975 : 88-89), apparait comme étant soustrait au contenu de l'assertion quoique présumé évident sur la base d'un savoir tenu pour acquis.

186 Comme présence dissimulée de segments discursifs antérieurs, le préconstruit ${ }^{17}$ transparaît dans le discours de l'énonciateur sous la forme de structures particulières comme la construction relative, la détermination, la nominalisation (Paveau 2006: 68). Ainsi, la nominalisation supposant la préconstruction d'un procès, comme le cas de « La baisse des effectifs » correspondant à « Les effectifs ont baissé » ou l'actualisation induite par l'article indéfini, pour le syntagme une fleur, par exemple, marquant la préconstruction d'un ensemble d'objets (Neveu $2004: 239-240$ ), cas particulier de ce que nous avons assimilé à la notion de hors-champ relatif. Dans cette optique, le marqueur prépositionnel sur (par opposition à la préposition à) pourrait être vu, de prime abord, comme l'indice de la présence d'un discours déjà produit. Or l'emploi situatif sur+ville ne semble, comme nous l'avons déjà signalé pour le présupposé, lié à une inférence stable : 
en réalité, cet emploi induit un ensemble non déterminé de virtualités, un hors-champ absolu.

En outre, le préconstruit se trouve lié à la notion de construction antérieure au moment de l'énonciation: «ça parle avant, ailleurs et indépendamment» du discours qui s'énonce. De ce point de vue, tandis que le préconstruit renvoie au "déjà dit» de l'interdiscours, le hors-champ absolu se rattache au «non vu» comme partie de l'énonciation. Ce non perçu / non montré concerne non seulement la catégorie du précédent mais intègre le synchrone et l'ultérieur (dans l'ordre du possible), introduisant, dans le cas qui nous occupe, une spatialisation évanescente : c'est l'introduction du temporel et du notionnel dans le cadre du tout dépassant le domaine de la spatialité.

\section{Conclusion}

Dans les pages qui précèdent, nous avons analysé l'essor d'un emploi innovant, celui de sur comme marqueur situatif devant les noms de ville, que nous avons décrit, dans un premier temps, à l'aide des concepts élaborés par Claude Vandeloise en sémantique prépositionnelle.

189 Cette caractérisation a été suivie d'une approche en termes de scène verbale, avec une attention particulière sur les opérations de cadrage, que nous avons distinguées du fenêtrage d'attention en ce que la détermination d'un plan (à échelle variable), suppose une tension, exploitable à des fins heuristiques, entre champ et hors-champ. Cette dernière notion a été employée pour une modélisation de la concurrence à / sur devant les toponymes urbains : l'emploi de la préposition sur s'est avéré intégrer une composante d'instabilité et de virtualité que nous avons rapportée au concept deleuzien de hors-champ absolu, comme ouverture sur une spatialité intégrant le temporel (dans le bornage et le provisoire) et le notionnel (dans l'exception et la non coïncidence).

190 La description en termes de hors champ (sous sa forme relative ou absolue) reste, pour l'instant, non formalisée. Cependant, même au stade d'ébauche, elle nous a permis de traiter un phénomène, celui du sémantisme prépositionnel divergent entre à et sur devant les noms de ville, en dépassant le cadre de ce qui est explicitement codé et en traitant les prépositions comme des déclencheurs d'inférence.

\section{BIBLIOGRAPHIE}

Brunot, F. 1953. La pensée et la langue. Méthode, principes et plan d'une théorie nouvelle du langage appliquée au français. Paris, Masson et Compagnie Editeurs.

Brunot, F. et Bruneau, C. 1956 [1933]. Précis de grammaire historique de la langue française. Paris, Masson et Cie.

Cadiot, P. 1991. De la grammaire à la cognition : la préposition POUR. Paris, Éditions du CNRS. 
Cadiot, P. 1997a. « Les paramètres de la notion de préposition incolore », Faits de Langues 9, pp. 127-134.

Cadiot, P. 1997b. Les prépositions abstraites en français. Paris, Armand Colin.

Cadiot, P. et Visetti, Y. -M. 2001. Pour une théorie des formes sémantiques. Motifs, profils, thèmes. Paris, Presses Universitaires de France.

Cervoni, J. 1991. La préposition. Étude sémantique et pragmatique. Paris, Duculot.

Charaudeau, P. 1992. Grammaire du sens et de l'expression. Paris, Hachette.

Col, G. et Victorri, B. 2007. « Comment formaliser en linguistique cognitive ? Opération de fenêtrage et calcul du sens temporel », Corela, Numéros spéciaux, « Cognition, discours, contextes ». Disponible sur : http://edel.univ-poitiers.fr/corela/document.php?id=1598 Colin, J. -P. 1994. Dictionnaire des difficultés du français. Paris, Dictionnaires Le Robert.

De Boer, C. 1926. Essai sur la syntaxe moderne de la préposition en français et en italien, Paris, Honoré Champion.

De Vega, M. et Rodrigo, M. -J. 1997. « Les représentations topologiques dans le traitement des descriptions spatiales » in Denis, M. (éd.), Langage et cognition spatiale. Paris, Masson, pp. 51-68.

Delattre, J. 2003. «Contre 'sur' », La Gazette n 113, p. 13.

Deleuze, G. 1983. Cinéma 1. L'image-mouvement. Paris, Les Éditions de Minuit.

Deleuze, G. 1985. Cinéma 2. L'image-temps. Paris, Les Éditions de Minuit.

Ducrot, O. 1984. Le dire et le dit. Paris, Les Éditions de Minuit.

Engel, P. 1994. Introduction à la philosophie de l'esprit. Paris, Éditions La Découverte.

Fauconnier, G. 1984. Espaces mentaux. Aspects de la construction du sens dans les langues. Paris, Editions de Minuit.

Franckel, J. -J. et Paillard, D. 2007. Grammaire des prépositions, Tome 1. Paris, Éditions Ophrys.

Girodet, J. 1997. Dictionnaire Bordas des pièges et difficultés de la langue française. Paris, LarousseBordas.

Gosselin, L. 1996. Sémantique de la temporalité en français.Louvain-la-Neuve, Duculot.

Gosselin, L. 2005. Temporalité et modalité. Bruxelles, De Boeck \& Larcier, Éditions Duculot.

Gougenheim, G. 1938. Système grammatical de la langue française. Paris, A. Routeix-D'artrey.

Gougenheim, G. 1972 [1952]. Les Mots Français dans l'histoire et dans lavie, Tome I. Paris, A. et J.

Picard.

Goyens, M., Lamiroy, B., Melis, L. 2002. « Déplacement et repositionnement de la préposition à en français ", Linguisticae Investigationes, 25 / 2, pp. 275-300.

Grevisse, M. 1980. Le Bon Usage. Paris-Gembloux, Duculot.

Habel, C. 1997. « Discours et représentations spatiales dans la description de plans » in Denis, M. (éd.), Langage et cognition spatiale. Paris, Masson, pp. 103-125.

Hernández, P. 2006. Effets de focalisation et de cadrage dans la mise en images de la localisation et de la configuration. Éléments pour une sémantique des prépositions spatiales en français au départ de l'espagnol. Prépositions françaises à - en - dans - chez - sur. Prépositions espagnoles a - en - sobre. Thèse de Doctorat, Université de Rouen. 
Herskovits, A. 1981. « On the spatial uses of prepositions in English », Linguistica Investigationes V/ 2, pp. 303-327.

Jackendoff, R. 1983. Semantics and cognition. Cambridge, M.I.T.

Langacker, R. W. 1987. Foundations of cognitive grammar. Vol. 1 Theoretical Prerequisites. Standford University Press.

Le Bidois, G. et Le Bidois, R. 1971. Syntaxe du Français Moderne. Ses fondements historiques et psychologiques, Tome II. Paris, Editions A. et J. Picard.

Mauger, G. 1968. Grammaire pratique du français d'aujourd'hui. Langue parlée, langue écrite. Paris, Hachette.

Melis, L. 2003. La préposition en français. Paris, Ophrys.

Neveu, F. 2004. Dictionnaire des sciences du langage. Paris, Armand Colin.

Paveau, M. -A. 2006. Les prédiscours. Sens, mémoire, cognition. Paris, Presses Sorbonne Nouvelle.

Pêcheux, M. 1975. Les vérités de La Palice. Linguistique, sémantique, philosophie. Paris, Librairie François Maspero.

Péchoin, D. et Dauphin, B. 1998. Dictionnaire des difficultés du français d'aujourd'hui. Paris, LarousseBordas.

Pottier, B. 1962. Systématique des éléments de relation. Paris, Librairie Klincksieck.

Ruwet, N. 1982. Grammaire des insultes et autres études. Paris, Editions du Seuil.

Sartre, J. -P. 1940. L'imaginaire. Paris, Gallimard.

Slakta, D. 1990. « Le "bon" génie de la langue et le rouge à lèvres », Europe, 738, 92-103.

Spang-Hanssen, E. 1963. Les prépositions incolores du français moderne. Copenhague, G.E.C. Gads Forlag.

Talmy, L. 1983. « How language structures space» in Pick, H. \& Acredolo, L. (eds). Spatial Orientation: Theory, Research and Application. New York, Plenum Press, pp. 225-282.

Talmy, L. 2000. Toward a Cognitive Semantics. Volume 1. London, Cambridge, The MIT Press.

Vandeloise, C. 1986. L'espace en français. Sémantique des prépositions spatiales. Paris, Editions du Seuil.

Vandeloise, C. 1987. « La préposition À et le principe d'anticipation », Langue Française 76, pp. 77-111.

Vandeloise, C. 1988. «Les usages spatiaux statiques de la préposition à », Cahiers de Lexicologie 53 Vol. 2, pp. 119-148.

Vandeloise, C. 1990. «Les frontières entre les prépositions sur et à », Cahiers deGrammaire 15, pp. 157-164.

Victorri, B. 1997. « La polysémie : un artefact de la linguistique? ». Revue de Sémantique et Pragmatique 2. Orléans, Université d'Orléans, pp .41-62.

Victorri, B. 1999. « Le sens grammatical». Langages136. Paris, Larousse, pp .85-105.

Victorri, B., Fuchs, C. 1996. La polysémie. Construction dynamique du sens. Paris, Hermès.

Von Wartburg, W. et Zumthor, P. 1973. Précis de syntaxe du français contemporain. Suisse, Editions Francke Berne. 
Wagner, R. L. et Pinchon, J. 1962. Grammaire du français classique et moderne. Paris, Librairie Hachette.

Weinrich, H. 1989. Grammaire textuelle du français, traduit par Gilbert Dalgalian et Daniel Malbert. Paris, Les Éditions Didier.

\section{ANNEXES}

1. Signalons cependant que la composante descriptive ne disparaît pas tout à fait. Si la préposition à peur être conçue comme a-descriptive, ce n'est pas le cas de sur qui, dans ses emplois locatifs conserve comme restriction de sélection l'allusion à un support.

Paul est à la plage / sur la plage

Paul est à l'école / ? sur l'école

Paul est au cinéma / ? sur le cinéma

Dans le cas de la plage, l'alternance entre à et sur n'entraîne pas de blocages majeurs pour l'interprétation de ces deux énoncés comme indicateurs de localisation. En revanche, avec des sites fermés (école, cinéma) les énoncés localisateurs en sur génèrent des doutes et l'alternance à-sur ne se fait pas sans interprétations divergentes : l'idée de support véhiculée par sur induit des configurations considérées comme anormales.

Cependant, force est de reconnaitre que des expressions telles que « le nombre des malades sur l'hôpital » dans les lèvres d'un correspondant de TV5 ou même certaines formulations des Forums sur Internet (Yahoo France le $1^{\mathrm{er}}$ juin 2009) laissent présager une évolution (nous transcrivons la graphie d'origine) : « il est obstétricien et qu'il est sur l'hôpital où je vais accoucher ", "vu qu'il y a peu qu'il est sur l'hôpital il en a remplacé un qui était génial » (14 occurrences d'énoncés en sur contre 25000 avec la préposition à). Début d'alternance à l'école / sur l'école (67 vs 12 700) avec des formulations telles que « il est sur l'école maternelle Lezay Mamésia depuis février 2006 », « Quand il est sur l'école en bus le matin, il me donne... »

2. Il ne s'agit pas, bien entendu, de confondre les emplois des deux relateurs sur lesquels pèsent des contraintes de sélection :

Le chien est sur le lit vs ${ }^{*}$ Le chien est au lit

La tasse est sur la table vs *La tasse est à la table

Paul est sur la plage vs Paul est à la plage

3. En moindre mesure devant les noms de pays, phénomène que nous n'analyserons pas ici - « À l'affreux « J'habite sur Paris » ou « sur le X ${ }^{\text {ème }}$ arrondissement » répond maintenant un «J'habite sur France mais je travaille sur Suisse » (sic ! Phrase entendue en Haute-Savoie) » (Delattre 2003).

4. Claude Vandeloise (1987) souligne que la préposition à peut être utilisée dans les mêmes circonstances que le relateur pour, usage qui ne s'est imposé que tardivement (Baudouin part pour Bruxelles = Baudouin part à Bruxelles). Pour G. Mauger (1968 : 393) c'est la ponctualité de la préposition à qui explique le tour partir à Paris où partir prend le sens de aller, au lieu de son sens premier, semettre en route. Le Bidois et Le Bidois (1971: 709) soulignent que ce « solécisme » a fait son chemin y compris dans la langue littéraire 
(« La femme partait à la Schola apprendre le contrepoint », Proust, Guermantes, 30) et considèrent que, lorsqu'on cherche à indiquer non pas le but du voyage mais son résultat, c'est-à-dire l'atteinte de la destination, il serait acceptable d'employer le relateur à et de dire « Il est parti à Paris (pour) faire des achats ».

5. Selon Grevisse (1980 : 1185), devant un nom de ville — surtout devant les noms des villes anciennes du bassin de la Méditerranée, affirme Gougenheim (1972 [1952] : 306) —, on employait au Moyen Age et encore au XVIIe siècle la préposition en comme réponse à la question où ? «Et li roy et la royne entrèrent en Callais » (Froissart, Cuuvres, t. V, p. 216) "J'écrivis en Argos » (Racine, Iphigénie, I, 1). Cependant, cet emploi a bientôt été concurrencé par celui de à de manière que les prépositions ont été partagées selon la règle à+ville, en+pays, norme établie par Meigret, puis par Ramus et précisée ensuite par Maupas et par Dupleix. Ainsi, en Avignon fait allusion à l'état papal d'Avignon (intégré à la France par l'Assemblée Nationale en 1791) ou même à la région d'Avignon. Mais, dès qu'il s'agit de la ville, c'est à Avignon qui est conforme à la règle. "Nous ne sommes pas à Avignon mais à Rivray » (H. de Régnier, LesVacances d'un Jeune Homme sage, p. 269). Autrement dit, la règle à+ville s'applique quelle que soit l'initiale du nom de la ville en question. L'expression en Avignon pour désigner la ville n'est qu'une variante stylistique de à Avignon visant à donner une couleur méridionale à la phrase sous l'influence du provençal : « Avec les noms de ville, en a cessé de se dire depuis le XVIIe s. [...] En Avignon est un provençalisme dont s'amuse A. Daudet » (Brunot 1953 : 425). Cet usage s'est étendu, chez certains écrivains, à d'autres villes dont le nom commence par A (Alger, Arles, Aix, Amiens, etc.) très probablement pour éviter l'hiatus: « Il s'en faut de tout qu'on soit 'miéterran' à Rome, à Gênes, à Valence et Madrid, à Bucarest ou à Fiume qu'enArles, enAix ou au Martigne » (A. Suarès, Vues sur l'Europe, p. 88, cité par Grevisse).

6. Les chiffres sont fournis à titre indicatif et ne peuvent être pris comme donnée scientifique car les certaines séquences apparaissent plus d'une fois dans les résultats fournis par le moteur de recherche. Par ailleurs, nous n'avons pas procédé à un dépouillement systématique des résultats, notre objectif étant tout simplement d'observer une tendance.

7. Disons, à titre indicatif, qu'une recherche, en cooccurrence avec le verbe être sur Google le 27 mai 2008 a donné comme résultat une proportion inverse : 6300 cooccurrences avec la préposition à vs 6450 avec le relateur sur.

8. Palmer, S. E. 1978. "Fundamental aspects of cognitive representations" in Rosch, E \& Lloyd, B. B. (éds). 1978. Cognition and categorization. Hillsdale, Erlbaum, pp. 259-303.

9. Husserl, E. éd. 1964. Leçons pour une phénoménologie de la conscience intime du temps. Paris, PUF.

10. S'il est possible de parler d'images visuelles ou auditives, c'est la référence à la vision qui semble s'imposer dans l'emploi usuel du terme image.

11. La perception pose son objet comme existant; la conscience imageante enferme, elle aussi, un acte positionnel : l'objet peut être considéré comme inexistant, absent, existant ailleurs ou il peut être neutralisé par une suspension de croyance (Sartre 1940 : 24). Dans le cas particulier du souvenir, proche de l'image, l'objet n'est pas posé comme donnéabsent mais comme donné-présent au passé (1940 : 230). 
12.Sont donc exclues des réflexions qui suivent les représentations par des moyens physiques (dessin, peinture, photo, etc.) Nous y aurons recours en 2.6. en tant qu'analogon des images mentales.

13. Wittgenstein, L. 1961. Tractatus Logico-philosophicus, trad. Klossowski. Paris, Gallimard, § 2.1. que nous citons d'après Gosselin (1996: 84).

14. Heidegger, M. 1985. Les problèmes fondamentaux de la phénoménologie, trad. J.-F. Courtine. Paris, Gallimard, p. 252, que nous citons d'après Gosselin (1996 : 84).

15. Nous reproduisons l'orthographe et la ponctuation des formulations d'origine.

16. Remarquons que ce dernier énoncé, tout en posant l'existence de deux espaces parallèles n'introduit pas de disjonction notionnelle.

17. La même dénomination est donnée par J. -B. Grize aux croyances individuelles en tant qu'acquis en fonction de l'environnement collectif. Les préconstruits culturels (PCC), proches de la compétence encyclopédique, participent à l'interprétation du discours (Grize, J. -B. 1998. « Logique naturelle, activité de schématisation et concept de représentation », Cahiers depraxématique 31, Montepellier, Praxiling, pp. 115-125 in Paveau, M. -A. 2006 : 73).

\section{RÉSUMÉS}

Inscrit dans le cadre des réflexions sur la scène verbale, le présent article traite du sémantisme de la préposition sur en emploi situatif devant les noms de ville, en concurrence avec la préposition à. Notre étude comporte deux volets. Le premier concernera le sémantisme prépositionnel. Après une présentation générale de la spatialité suggérée par les prépositions à et sur, nous concentrerons notre analyse sur les particularités de l'emploi sur+ville. Le deuxième volet abordera le traitement de la spatialité selon un phénomène de cadrage : la tension entre champ et hors-champ. Enfin, les deux volets se rejoindront pour modéliser l'emploi de sur+ville en termes de hors-champ absolu

In the context of scène verbale (verbal scene), this paper deals with the semantics of the preposition sur as a locative marker in competition with preposition $\grave{a}$ in phrases including names of cities. Our study comprises two sections. The first one addresses the semantics of both prepositions. After a general presentation of the spatiality suggested by the prepositions $\grave{a}$ and sur, we analyze the case of utterances such as sur+city. The second focuses on the spatiality according to a phenomenon of visual framing: the tension between champ and hors-champ (in frame vs. out of frame). Finally, we propose a model in terms of hors-champ absolu (absolute out of frame). This device enables us to describe the semantics of sur+city.

\section{INDEX}

Keywords : configuration, framing, inference, localization, out of frame, preposition, semantics, verbal scene

Mots-clés : cadrage, configuration, hors-champ, inférence, localisation, préposition, scène verbale, sémantique 


\section{AUTEUR}

\section{PATRICIA C. HERNÁNDEZ}

Instituto de Enseñanza Superior en Lenguas Vivas « Juan R. Fernández », Instituto Superior del Profesorado « Dr. Joaquín V. González », Buenos Aires 